\title{
Alternative Technique for the Constrained Variational Problem Based on an Asymptotic Projection Method: II. Applications to Open-Shell Self-Consistent Field Theory
}

\author{
Vitaly N. Glushkov(®), Nikitas I. Gidopoulos, and Stephen Wilson
}

\begin{abstract}
The problem of the orthogonality of the closed- and open-shell orbitals in self-constistent field (SCF) theory are reviewed. An easily implemented asymptotic projection method for talking orthogonality constraints into account is used to develop an alternative open-shell SCF approach for ground and excited states. Variational derivation of the open-shell Hartree-Fock equations is described which does not involve off-diagonal Lagrangian multipliers. The possibilities of designing a well-defined, open-shell many-body perturbation theory using the orbitals of the asymptotic projection method is demonstrated by means of calculations of ground and excited state energies using the different levels of approximation. We also focus on the development of alternative computational strategies capable of optimizing relatively small distributed basis sets with respect to the positions and exponents of the basis functions.
\end{abstract}

Keywords: open-shell system, orthogonality constraints, self consistent field, excited state, many body perturbation theory

\footnotetext{
V.N. Glushkov

Department of Physics, National University, Dnepropetrovsk, 49050, per.Nauchny 13, Ukraine, e-mail: v_n_glushkov@yahoo.com

N.I. Gidopoulos

ISIS Facility, Rutherford Appleton Laboratory, Chilton, Didcot, Oxon, OX11 0QX, England, UK, e-mail: n.gidopoulos@rl.ac.uk

S. Wilson

Physical \& Theoretical Chemistry Laboratory, University of Oxford, South Parks Road, Oxford OX1 3QZ, England;

Faculty of Mathematics, Physics and Informatics, Comenius University, 84215 Bratislava, Slovakia, e-mail: quantumsystems@gmail.com
} 


\section{Introduction}

Open-shell SCF theory is one of the most elegant techniques of contemporary quantum chemistry. Computational methods based on open-shell theory provide well-established tools which are applied routinely in modern molecular electronic structure studies (see, e.g. [1-14] and references therein). Today, open-shell methodology is available in many of the quantum chemical program packages, such as GAUSSIAN [23] and GAMESS [24], and is exploited in many practical applications.

In open-shell SCF theory, the optimal orbitals $\varphi_{i}, i=1,2, \ldots, N$ satisfy equations of the following form:

$$
\begin{gathered}
F_{i}\left|\varphi_{i}\right\rangle=\sum_{j}\left|\varphi_{j}\right\rangle \theta_{j i} \\
\theta_{i j}=\theta_{j i}^{*}
\end{gathered}
$$

where the $F_{i}$ are Fock operators and the $\theta_{i j}$ are Lagrangian multipliers, which arise from the orthogonality constraints

$$
\left\langle\varphi_{i} \mid \varphi_{j}\right\rangle=\delta_{i j}
$$

The Fock operators are functionals of $\left\{\varphi_{i}\right\}$ and so the above equations are solved iteratively until self-consistency is attained. If a unitary transformation between the $\varphi_{i}$ is possible without changing the total energy expectation, then some or all of conditions (1) can be fulfilled. This is a case, for example, in closed-shell SCF theory.

When describing open-shell systems, conventional approaches give rise to Hartree-Fock type equations which involve different Fock operators for the orbitals with the same spin. This results in off-diagonal Lagrange multipliers which couple the closed- and open-shells and which cannot be eliminated by a suitably chosen unitary transformation. Many studies have been devoted to the orthogonality problem for the closed- and open-shell orbitals [1-10]. In his seminal 1960 paper, Roothaan [1] proposed the elegant coupling operator formalism to overcome this difficulty. Considerable progress has been made in understanding the nature of the coupling operator method (see, e.g., [2-8], [11]) and useful computational experience has been accumulated in solving the restricted open-shell Hartree-Fock (ROHF) equations (see, e.g., [12-14]). Recent investigations have made it possible to find a canonical form of the open-shell Fock operator, which leads to useful relations between the open-shell orbital energies and the ionization potentials [17]. An excellent description of a history and evolution of the SCF theory for open-shell systems can be found in the review by Carbo and Riera [14] and references therein. At the present time, the coupling operator based methods, first proposed by Roothaan in 1960 , form the basis of the most widely used computational procedure in studies of open-shell systems.

The choice of zero-order Hamiltonian is crucial to the success of perturbation approaches to the correlation problem and this choice is not as straightforward for open-shell systems as it is in the case of closed-shell species. A number of open-shell versions of many-body perturbation theory have been developed over 
the years beginning with the work of Hubač and Čársky in 1980 [18], which was extended through fourth order by one of the present authors [19]. Much work on open-shell systems has concentrated on states of high spin. Of course, the description of arbitrary open-shell systems requires the use of a multireference perturbation expansion.

The Roothaan coupling operator formalism does not readily lend itself to a well-defined perturbation theory for describing the correlation effects in arbitrary open-shell systems [20-25]. In recent years, there has been renewed interest in the open-shell methods fuelled by the desire to define a cost-effective many-body perturbation theory based on restricted open-shell Hartree-Fock wave functions. For general open-shell systems, it can be difficult to devise a zero-order Hamiltonian $H^{(0)}$ for which the wave function has a simple form and is an eigenfunction of the total spin operator $\mathbf{S}^{2}$ [26]. Within the framework of the Roothaan coupling operator approach, there is no unique way of choosing a reference Hamiltonian, $H^{(0)}$, with respect to which a perturbation expansion for correlation effects can be developed. Several proposals have been made for open-shell many-body perturbation theory expansions (or open-shell Møller-Plesset-like perturbation theory (MPPT)) based on a reference from the ROHF formalism $[22,23]$ or the unrestricted Hartree-Fock (UHF) formalism [20,21]. (It is well-known that the UHF formalism has a number of serious deficiencies, such as unphysical features in calculated potential energy curves.) These approaches to open-shell many-body perturbation theory differ primarily in the definition of the reference hamiltonian $H^{(0)}$. It is well established that the success or failure of a particular perturbation theory expansion is largely determined by the choice of the reference Hamiltonian, $H^{(0)}$. Ambiguities in the definition of the zero-order operator cannot be regarded as a desirable element of any perturbation theory.

In Sect. 2, we shall demonstrate that the method of asymptotic projection, which was reviewed in paper 1 [27], can be used to avoid ambiguities in the definition of zero-order operators for use in developing perturbation expansions for correlation effects. In previous work [28-38], the asymptotic projection method has been shown to be useful tool for solving quantum chemical problems which can be formulated in terms of an eigenvalue problem with orthogonality restrictions, i.e. the constrained variational problem reviewed in paper 1 [27].

Another aspect of SCF theory which deserves more detailed investigation is the study of electronically excited states, and especially excited states of the same spin and spatial symmetry as the ground or some lower-lying state. Indeed, Hartree-Fock calculations for electronically excited states cannot be considered routine. In particular, remembering that the ground and excited states are often of quite different character, it is desirable to use different basis sets for different states. "The desirability of using different basis sets for different states" was pointed out by Shull and Löwdin [39] as long ago as 1958. Such an approach provides a compact and accurate representation of excited state wave functions. Today, the most commonly used approaches to the study of excited states are based on multireference techniques, including configuration interaction, the multiconfigurational self-consistent field method and its "complete active space" variant designated CASSCF, multireference 
perturbation theory and multireference coupled cluster expansions. These methods are indispensable in studies of systems for which single-configuration methods cannot be applied - for example, when the weight of the Hartree-Fock configuration in the wave function of the full configuration interaction expansion is less than $\sim 0.9$ [40]. However, in cases where a multireference approach is necessary, it is clear that the orbitals of a single-configuration, together with a basis set that has been specifically optimized for a given excited state, will prove more appropriate for the development of many-body correlation methods than orbitals expanded in a basis set constructed for the ground state. Furthermore, progress in excited state SCF theory might be expected to aid the development of density functional theory for excited states.

In Sect. 3, we shall consider the use of the asymptotic projection method in excited state SCF calculations. The many-body Møller-Plesset-like perturbation theory based on optimal orbitals generated by the SCF-asymptotic projection method is the subject of Sect. 4. We shall demonstrate that, unlike existing open-shell perturbation theory formalisms, our alternative methodology can be easily extended to excited states and, thus, facilitate the computation of a large part of the correlation energy in a rather simple way. Our concluding remarks are given in Sect. 5.

\section{Restricted Open-Shell Wave Functions and the Asymptotic Projection Method}

In this section, we review an alternative to the Roothaan's open-shell method that does not involve off-diagonal Lagrange multipliers. We develop the asymptotic projection-SCF formalism to construct a single open-shell Slater determinant from which a well-defined, open-shell, many-body Møller-Plesset-like perturbation theory (MPPT) can be performed for both the ground and excited states.

\subsection{Modified Open-Shell Hartree-Fock Equations for Ground States}

\subsubsection{Notation, Conventions and Restrictions}

In this subsection, we shall consider systems for which the total SCF wave function can be written as a sum of several antisymmeterized products, each of which consists of a product of doubly occupied orbitals $\varphi_{k}^{c}$, the so-called core orbitals or closedshell set, and singly occupied orbitals $\varphi_{m}$, the valence orbitals or open-shell set. To clarify our alternative technique, i.e. asymptotic projection, we shall restrict our attention to open-shell systems for which the expression for the energy expectation value can be partitioned as follows:

$$
E=E^{c}+E^{o}+E^{c o}
$$


where the first term on the right-hand side is the closed-shell energy, the second term is the open-shell energy, and the last term arises from the interaction between the closed and open shells. This is a case considered in Roothaan's seminal article [1], for which the energy components have the form

$$
\begin{gathered}
E^{c}=2 \sum_{k} h_{k k}+\sum_{k, l}\left(2\left\langle\varphi_{k}\left|J_{l}\right| \varphi_{k}\right\rangle-\left\langle\varphi_{k}\left|K_{l}\right| \varphi_{k}\right\rangle\right) \\
E^{o}=f\left[2 \sum_{m} h_{m m}+f \sum_{m, n}\left(2 a\left\langle\varphi_{m}\left|J_{n}\right| \varphi_{m}\right\rangle-b\left\langle\varphi_{m}\left|K_{n}\right| \varphi_{m}\right\rangle\right)\right]
\end{gathered}
$$

and

$$
E^{c o}=2 f \sum_{k, m}\left(2\left\langle\varphi_{k}\left|J_{m}\right| \varphi_{k}\right\rangle-\left\langle\varphi_{k}\left|K_{m}\right| \varphi_{k}\right\rangle\right)
$$

In these expressions, $a, b$, and $f$ are numerical constants depending on the particular state under consideration, $h_{k k}=\left\langle\varphi_{k}|h| \varphi_{k}\right\rangle$ where $h$ is the one-electron operator describing the kinetic energy of an electron and its interactions with the nuclei, $J_{k}$ and $K_{k}$ are commonly called the Coulomb and exchange operators, which are defined as

$$
\left\langle\varphi_{i}\left|J_{j}\right| \varphi_{i}\right\rangle=\left(\varphi_{i} \varphi_{i} \mid \varphi_{j} \varphi_{j}\right)=\int \varphi_{i}^{*}(1) \varphi_{j}^{*}(2) \frac{1}{r_{12}} \varphi_{i}(1) \varphi_{j}(2) d V_{1} d V_{2}
$$

and

$$
\left\langle\varphi_{i}\left|K_{j}\right| \varphi_{i}\right\rangle=\left(\varphi_{i} \varphi_{j} \mid \varphi_{i} \varphi_{j}\right)=\int \varphi_{i}^{*}(1) \varphi_{i}^{*}(2) \frac{1}{r_{12}} \varphi_{j}(1) \varphi_{j}(2) d V_{1} d V_{2},
$$

respectively. Following Roothaan [1], we reserve the indices $k, l$ and $m, n$ for the closed-shell and open-shall orbitals, respectively, and the indices $i, j$ for orbitals of either set.

\subsubsection{Orthogonality Constraints in the Restricted Open-Shell SCF Formalism}

In the following discussion, we will be concerned with the orthogonality constraints which are to be imposed on the orbitals. Such constraints can be divided into two types. The first type of constraints are the orthogonality conditions within each of the orbital sets: $\left\{\varphi_{k}^{c}\right\},\left\{\varphi_{m}\right\}$, i.e.

$$
\begin{gathered}
\left\langle\varphi_{k}^{c} \mid \varphi_{l}^{c}\right\rangle=\delta_{k l} \\
\left\langle\varphi_{m} \mid \varphi_{n}\right\rangle=\delta_{m n} .
\end{gathered}
$$

These constraints are usually incorporated by introducing the Lagrange multipliers $\left\{\theta_{k l}^{c}\right\}$ and $\left\{\theta_{m n}^{o}\right\}$. The corresponding matrices can always be transformed to diagonal form by appropriate unitary transformations. The second type of constraints are the orthogonality conditions between the closed-shell and the and open-shell orbitals, i.e.

$$
\left\langle\varphi_{k}^{c} \mid \varphi_{m}\right\rangle=0
$$


It is from the condition (6) that the problem of off-diagonal Lagrange multipliers arises. In the present study, we shall use the asymptotic projection technique to handle these orthogonality constraints rather than the conventional coupling operator methods introduced by Roothaan. It is convenient when using the asymptotic projection method to rewrite Eq. (6) in two related symmetrical forms which are convenient for carrying out the variations; namely,

$$
\sum_{k}\left\langle\varphi_{k}^{c}\left|P_{o}\right| \varphi_{k}^{c}\right\rangle=0
$$

and

$$
\sum_{m}\left\langle\varphi_{m}\left|P_{c}\right| \varphi_{m}\right\rangle=0
$$

where

$$
P_{c}=\sum_{k}\left|\varphi_{k}^{c}\right\rangle\left\langle\varphi_{k}^{c}\right|
$$

and

$$
P_{o}=\sum_{m}\left|\varphi_{m}\right\rangle\left\langle\varphi_{m}\right|
$$

are the orthoprojectors on the subspaces spanned by the closed-shell and open-shell orbitals, respectively.

It should be noted that each term in the sum (7) [or (8)] is nonnegative, therefore, the requirement (7) [or (8)] is exactly equivalent to the orthogonality conditions (6). Thus, when implementing a variational principle, as we shall demonstrate in the next subsection, we may apply the constraint (7) [or (8)] instead of using (6).

\subsubsection{Variational Derivation of the Open-Shell Hartree-Fock Equations}

We start from the stationary condition for the total energy given in Eq. (2) subject to the constraints (4), (5) and (7).

Let us now define the functional

$$
L=E-2 \sum_{k, l} \theta_{k l}^{c}\left\langle\varphi_{k}^{c} \mid \varphi_{l}^{c}\right\rangle-2 \sum_{m, n} \theta_{m n}^{o}\left\langle\varphi_{m} \mid \varphi_{n}\right\rangle+\lambda \sum_{k}\left\langle\varphi_{k}^{c}\left|P_{o}\right| \varphi_{k}^{c}\right\rangle
$$

where $\theta_{m n}^{c}, \theta_{m n}^{o}$ and $\lambda$ are Lagrange multipliers introduced to take account of the orthogonality constraints.

The stationary condition $\delta L=0$ takes the form

$$
\delta L=\delta\left\{E-2 \sum_{k, l} \theta_{k l}^{c}\left\langle\varphi_{k}^{c} \mid \varphi_{l}^{c}\right\rangle-2 \sum_{m, n} \theta_{m n}^{o}\left\langle\varphi_{m} \mid \varphi_{n}\right\rangle\right\}+\lambda \delta\left\{\sum_{k}\left\langle\varphi_{k}^{c}\left|P_{o}\right| \varphi_{k}^{c}\right\rangle\right\}=0 .
$$

After some manipulation, the variations in the first bracket of Eq. (12) yield the standard result:

$$
\sum_{k}\left\langle\delta \varphi_{k}^{c}\left|\left(F^{c}-\varepsilon_{k}^{c}\right)\right| \varphi_{k}^{c}\right\rangle+\sum_{m}\left\langle\delta \varphi_{m}\left|\left(F^{o}-\varepsilon_{m}^{o}\right)\right| \varphi_{m}\right\rangle+c . c .
$$


where c.c. denotes the complex conjugate and $\varepsilon_{k}^{c}$ and $\varepsilon_{m}^{o}$ are the diagonal elements of matrices $\theta_{k l}^{c}$ and $\theta_{m n}^{o}$, respectively.

In Eq. (13) $F^{c}$ and $F^{o}$ are Fock operators (or Fockians), whose explicit form depends on the precise form of the energy expression of the state under consideration. For example, for the expression (2), we have [1]:

$$
\begin{gathered}
F^{c}=h+\sum_{k}\left(2 J_{k}^{c}-K_{k}^{c}\right)+f \sum_{m}\left(2 J_{m}-K_{m}\right) \\
F^{o}=h+\sum_{k}\left(2 J_{k}^{c}-K_{k}^{c}\right)+f \sum_{m}\left(2 a J_{m}-b K_{m}\right)
\end{gathered}
$$

where $h$ is the one-electron operator describing the kinetic energy of an electron and its interaction with the nuclei. It can be shown [24] that the variations in the second bracket in Eq. (12) lead to the following expression

$$
\delta\left\{\sum_{k}\left\langle\varphi_{k}^{c}\left|P_{o}\right| \varphi_{k}^{c}\right\rangle\right\}=\sum_{k}\left\langle\delta \varphi_{k}^{c}\left|P_{o}\right| \varphi_{k}^{c}\right\rangle+\sum_{m}\left\langle\delta \varphi_{m}\left|P_{c}\right| \varphi_{m}\right\rangle+c . c .
$$

Combining Eqs. (13) and (14), the total variation in Eq. (12) can be written as follows:

$$
\delta L=\sum_{k}\left\langle\delta \varphi_{k}^{c}\left|\left(F^{c}+\lambda P_{o}-\varepsilon_{k}^{c}\right)\right| \varphi_{k}^{c}\right\rangle+\sum_{m}\left\langle\delta \varphi_{m}\left|\left(F^{o}+\lambda P_{c}-\varepsilon_{m}^{o}\right)\right| \varphi_{m}\right\rangle+c . c .
$$

In practice, the orbitals are approximated by means of some expansion in a finite one-particle basis set (the algebraic approximation is invoked), i.e.,

$$
\left|\varphi_{i}\right\rangle=P\left|\varphi_{i}\right\rangle=\sum_{q=1}^{Q} C_{i q}\left|\chi_{q}\right\rangle
$$

where $\mathrm{P}$ is an orthoprojector defined by a chosen basis set $\left\{\chi_{q}\right\}$. Using this expansion, the orbital variations can be written in the form

$$
\left|\delta \varphi_{i}\right\rangle=P\left|\delta \varphi_{i}\right\rangle+\sum_{a}\left(\partial_{a} P\right)\left|\varphi_{i}\right\rangle \delta \mu_{a}
$$

where $\mu_{a}, a=1,2, \ldots, \mathrm{A}$, represents the basis set parameters (i.e. the exponents and the positions). The first term in Eq. (16) corresponds to variations within the finitedimensional subspace spanned by the chosen basis set $\left\{\chi_{q}\right\}$, whereas the second term allows this subspace to be rotated within the Hilbert space of one-particle states to attain the deeper minimum with respect to the total energy.

Substituting Eq. (16) into Eq. (15) and taking into account the independence of the variations and their arbitrariness, we obtain the following equations:

$$
P\left(F^{c}+\lambda P_{o}-\varepsilon_{k}^{c}\right) P\left|\varphi_{k}^{c}\right\rangle=0
$$

and

$$
P\left(F^{o}+\lambda P_{c}-\varepsilon_{m}^{o}\right) P\left|\varphi_{m}\right\rangle=0
$$


According to the asymptotic projection methodology, the imposition of the condition $\lambda \rightarrow \infty$ in Eqs. (17) and (18) ensures the orthogonality between closed- and open-shell orbitals. The choice of $\lambda$, whose value determines the target accuracy for practical calculations, will be discussed below. Here it is worth noting that orbital energies will be shifted during the iterative solution of Eqs. (17) and (18). Therefore, after a solution has been obtained, these equations should be redefined as:

$$
\varepsilon_{k}^{c}=\left\langle\varphi_{k}^{c}\left|F^{c}\right| \varphi_{k}^{c}\right\rangle \quad \text { and } \quad \varepsilon_{m}^{o}=\left\langle\varphi_{m}\left|F^{o}\right| \varphi_{m}\right\rangle .
$$

The second term on the right-hand side of Eq. (16) leads to the equations for optimization of the basis set:

$$
\sum_{k}\left\langle\varphi_{k}^{c}\left|\left(\partial_{a} P\right) F^{c}\right| \varphi_{k}^{c}\right\rangle+\sum_{m}\left\langle\varphi_{m}\left|\left(\partial_{a} P\right) F^{o}\right| \varphi_{m}\right\rangle=0 .
$$

In Eq. (19) we took account of the fact that the term $\lambda P\left(\partial_{a} P\right) P$ vanishes for arbitrary $\lambda$. The left-hand side of Eq. (19) is the expression for the energy gradient with respect to the basis set parameters. This expression allows these parameters to be determined variationally.

\subsubsection{Correct Variational Conditions and Orbitals Based on Asymptotic Projection}

It is known that optimum set of orbitals must satisfy the correct variational conditions, which are equivalent to the generalized Brillouin's theorem [8]. There are two distinct types of variational conditions for optimal orbitals. The first type corresponds to the variational condition between virtual orbitals $\left\{\varphi_{a}\right\}$ and occupied $\left\{\varphi_{i}\right\}$ orbitals, i.e.

$$
\left\langle\varphi_{a}\left|F^{c}\right| \varphi_{k}^{c}\right\rangle=0
$$

and

$$
\left\langle\varphi_{a}\left|F^{o}\right| \varphi_{m}\right\rangle=0 .
$$

The second type of variational condition is that among occupied orbitals, i.e.

$$
\left\langle\varphi_{m}\left|\left(F^{c}-F^{o}\right)\right| \varphi_{k}^{c}\right\rangle=0
$$

From Eqs. (17) and (18) it is easy to show that the orbitals based on asymptotic projection obey these conditions. For example, multiplying Eq. (17) by $\left\langle\varphi_{m}\right|$ and Eq. (18) by $\left\langle\varphi_{k}^{c}\right|$ by taking into account the orthogonality of the limiting orbitals, we obtain

$$
\left\langle\varphi_{m}\left|F^{c}\right| \varphi_{k}^{c}\right\rangle=-\lambda\left\langle\varphi_{m} \mid \varphi_{k}^{c}\right\rangle
$$

and

$$
\left\langle\varphi_{k}^{c}\left|F^{o}\right| \varphi_{o}\right\rangle=-\lambda\left\langle\varphi_{k}^{c} \mid \varphi_{m}\right\rangle .
$$


Finally, by subtracting these equations and taking the Hermitian properties of operators $F^{c}$ and $F^{o}$ into account, we arrive at the second variational condition, Eq. (22). The relations (20) and (21) can be proved in a similar manner.

Thus, Eqs. (17) and (18) lead to an optimal set of orbitals. The energy expectation value supported by such orbitals is identical to that obtained by the Roothaan coupling operators formalism. However, when implemented in a finite basis set approach (the algebraic approximation), the coupling operator method deals with equations of fifth order with respect to the coefficients $C_{i q}$ in the two operator formalism and seventh order in the unique coupling operator formalism because of the use of exchange coupling operators $J_{o}$ and $K_{o}$ (see Eq. (19) in Roothaan's paper [1]) whereas the self-consistent field equations in the asymptotic projection technique, Eqs. (17) and (18), are cubic equations.

\subsection{Unrestricted Hartree-Fock (UHF) Formalism for Obtaining High-Spin Restricted Open-Shell Hartree-Fock (ROHF) Functions}

It is well known that a large class of open-shell systems can be described by a single Slater determinant. The open-shell Slater determinant $\Phi$ is built from orbitals $\varphi_{k}^{\alpha}, k=1,2, \ldots, n^{\alpha}$, associated with $\alpha$ spin and orbitals $\varphi_{k}^{\beta}, k=1,2, \ldots, n^{\beta}$, associated with $\beta$ spin. In addition, $n^{\alpha} \geqslant n^{\beta}$ and $n=n^{\alpha}+n^{\beta}$ is the total number of electrons, and $S=S_{z}$. As we mentioned above, in the traditional open-shell method there is a degree of arbitrariness in the Fock operators that leads to different forms of perturbation expansion for the correlation energy. These ambiguities can be avoided by using the UHF formalism in which we allow the spatial part of the $\alpha$ spin orbitals to differ from that of the $\beta$ spin orbitals. A spin purity constraint should be imposed on the spatial orbitals in order to eliminate spin contamination in the UHF function. This requirement is known [41] to be fulfilled if the occupied $\beta$ orbitals are a linear combination of occupied $\alpha$ orbitals, i.e. the $\beta$ set lies completely within the subspace defined by the $\alpha$ set. Below we shall see that this requirement leads to a restricted open-shell Hartree-Fock Slater determinant.

Now we have

$$
P^{\alpha} P^{\beta}=P^{\beta} P^{\alpha}=P^{\beta}
$$

with

$$
P^{\alpha}=\sum_{k}^{n^{\alpha}}\left|\varphi_{k}^{\alpha}\right\rangle\left\langle\varphi_{k}^{\alpha}\right| \quad \text { and } \quad P^{\beta}=\sum_{k}^{n^{\beta}}\left|\varphi_{k}^{\beta}\right\rangle\left\langle\varphi_{k}^{\beta}\right| .
$$

This allows us to rewrite the spin-purity requirement as the orthogonality constraint

$$
\sum_{k}^{n^{\beta}}\left\langle\varphi_{k}^{\beta}\left|Q^{\alpha}\right| \varphi_{k}^{\beta}\right\rangle=0
$$

where $Q^{\alpha}=I-P^{\alpha}$ is the orthoprojector on the subspace of the virtual $\alpha$ spin orbitals. 
Following the asymptotic projection methodology, the Euler equations for orbitals can be then derived from the stationary condition

$$
\delta L=\delta\left[E^{U H F}+\lambda \sum_{k}^{n^{\beta}}\left\langle\varphi_{k}^{\beta}\left|Q^{\alpha}\right| \varphi_{k}^{\beta}\right\rangle\right]=0 .
$$

Similarly using Eq. (16), variations of the orbitals can be divided into independent parts. For example, for the $\alpha$ orbitals we have

$$
\left|\delta \varphi_{i}^{\alpha}\right\rangle=P^{\alpha}\left|\delta \varphi_{i}^{\alpha}\right\rangle+\left(I-P^{\alpha}\right)\left|\delta \varphi_{i}^{\alpha}\right\rangle+\sum_{a}\left(\partial_{a} P\right)\left|\varphi_{i}^{\alpha}\right\rangle \delta \mu_{a}
$$

Energetically significant variations are described by the second and third terms in Eq. (24) because the first term does not lead to any change in the total energy since it is invariant to any orthogonal transformation of the orbitals associated with a given spin among themselves.

Substituting Eq. (24) into Eq. (23), after some manipulation, we arrive at the following set of equations, which determine the optimal orbitals (see Glushkov [24, 33] for more details):

$$
\begin{aligned}
& \lim _{\lambda \rightarrow \infty} P\left(F^{\alpha}-\lambda P^{\beta}-\varepsilon_{i}^{\alpha}\right) P\left|\varphi_{i}^{\alpha}\right\rangle=0, \quad i=1,2, \ldots, n^{\alpha}, \ldots, M \\
& \lim _{\lambda \rightarrow \infty} P\left(F^{\beta}+\lambda Q^{\alpha}-\varepsilon_{i}^{\beta}\right) P\left|\varphi_{i}^{\beta}\right\rangle=0, \quad i=1,2, \ldots, n^{\beta}, \ldots, M
\end{aligned}
$$

and equations for basis set optimization

$$
\sum_{k}^{n^{\alpha}}\left\langle\varphi_{k}^{\alpha}\left|\left(\partial_{a} P\right) F^{\alpha}\right| \varphi_{k}^{\alpha}\right\rangle+\sum_{k}^{n^{\beta}}\left\langle\varphi_{k}^{\beta}\left|\left(\partial_{a} P\right) F^{\beta}\right| \varphi_{k}^{\beta}\right\rangle=0 .
$$

In Eqs. (25-27) $F^{\alpha}$ and $F^{\beta}$ are the conventional UHF operators. It should be stressed that each of the additional term in (25) and (26) ensures spin purity, i.e.

$$
\widehat{\mathbf{S}}_{z}|\Phi\rangle=S|\Phi\rangle, \quad \widehat{\mathbf{S}}^{2}|\Phi\rangle=S(S+1)|\Phi\rangle
$$

but only both of the terms $\lambda P^{\beta}$ and $\lambda Q^{\alpha}$ in combination with the limit $\lambda \rightarrow \infty$ lead to an optimum set of orbitals satisfying the generalized Brillouin's theorem (see next section for further discussion). Note, that together both additional terms also lead to spatial parts of the $\alpha$ set which are identical to those of the $\beta$ set, but the corresponding orbital energies are different. The Slater determinant built from these orbitals gives a minimum of the total energy expectation value which is equivalent to that obtained by the Roothaan coupling operator method.

In concluding this section, we note that the Eq. (27) is the natural generalization of the equations for the optimization of the basis set for closed-shell systems. Indeed, Eq. (27) reduces to that for the optimization of the basis set for closed systems in the case $n^{\alpha}=n^{\beta}$ (see, [42-44]).

$$
\partial_{a} E=2 \sum_{i}\left\langle\varphi_{i}\left|\left(\partial_{a} P\right) F\right| \varphi_{i}\right\rangle=0
$$




\subsubsection{Generalized Brillouin's Theorem in Terms of Unrestricted Hartree-Fock Orbitals Based on Asymptotic Projection}

Below we shall show that the orbitals generated by Eqs. (25) and (26) in the limit $\lambda \rightarrow \infty$ satisfy the correct variational conditions i.e. the generalized Brillouin's theorem.

Without loss of generality, the full orbital space may be divided into a closedshell part $(c)$, an open-shell part $(o)$ and a virtual part $(v)$. According to the generalized Brillouin's theorem, we have $[7,8]$

$$
\langle\Phi|H| \Phi(i \rightarrow j)\rangle=0
$$

$\Phi(i \rightarrow j)\rangle$ are configurations which are singly excited with respect to $\Phi$. There are three types of well-defined singly excited configurations with the same multiplicity as $\Phi[7,8]$, viz.:

$$
\begin{gathered}
\Phi(c \rightarrow o)=\operatorname{det}|c \alpha o \beta o \alpha|, \\
\Phi(o \rightarrow v)=\operatorname{det}|c \alpha c \beta v \alpha|, \\
\Phi(c \rightarrow v)=N(\operatorname{det}|c \alpha v \beta o \alpha|+\operatorname{det}|v \alpha c \beta o \alpha|),
\end{gathered}
$$

in addition

$$
\Phi=\operatorname{det}|c \alpha c \beta o \alpha| .
$$

For the sake of simplicity, we denote only the spin-orbitals which take part in excitations in the determinants. $N$ is a normalization multiplier. The indices $c, o$ and $v$ mean closed-shell (doubly occupied), open-shell (singly occupied) and the virtual parts of the full orbital space, respectively. In terms of the unrestricted Hartree-Fock operators $F^{\alpha}$ and $F^{\beta}$, Brillouin's theorem can be written:

$$
\begin{array}{r}
\langle\Phi|H| \Phi(c \rightarrow o)\rangle=\left\langle\varphi_{i}^{\beta}\left|F^{\beta}\right| \varphi_{m}^{\beta}\right\rangle=0, \quad(m \in o, i \in c) \\
\langle\Phi|H| \Phi(o \rightarrow v)\rangle=\left\langle\varphi_{m}^{\alpha}\left|F^{\alpha}\right| \varphi_{a}^{\alpha}\right\rangle=0, \quad(m \in o, a \in v) \\
\langle\Phi|H| \Phi(c \rightarrow v)\rangle=\left\langle\varphi_{a}^{\alpha}\left|F^{\alpha}\right| \varphi_{i}^{\alpha}\right\rangle+\left\langle\varphi_{a}^{\beta}\left|F^{\beta}\right| \varphi_{i}^{\beta}\right\rangle=0, \quad(i \in c, a \in v) .
\end{array}
$$

Brillouin's theorem is obeyed for the set of orbitals satisfying Eqs. (25) and (26) in the limit $\lambda \rightarrow \infty$ as well as the 'traditional' orbitals obtained by the Roothaan approach. Indeed, multiplying Eq. (25) by $\left\langle\varphi_{a}^{\alpha}\right|$ and Eq. (26) by $\left\langle\varphi_{a}^{\beta}\right|$, we obtain

$$
\left\langle\varphi_{a}^{\alpha}\left|F^{\alpha}\right| \varphi_{i}^{\alpha}\right\rangle=\lambda\left\langle\varphi_{a}^{\alpha} \mid \varphi_{i}^{\beta}\right\rangle, \text { and }\left\langle\varphi_{a}^{\beta}\left|F^{\beta}\right| \varphi_{i}^{\beta}\right\rangle=-\lambda\left\langle\varphi_{a}^{\alpha} \mid \varphi_{i}^{\beta}\right\rangle \text {. }
$$

A constraint vector has been shown to tend to an eigenvector of the modified operator as $1 / \alpha$ (see Part I of this work [27]). Therefore, the limit $\lambda\left\langle\varphi_{a}^{\alpha} \mid \varphi_{i}^{\beta}\right\rangle, \lambda \rightarrow \infty$, exists and from Eq. (33) we can confirm that the condition (32) is satisfied. In the same manner, we can show that conditions (30) and (31) are also satisfied for the orbitals in the limit $\lambda \rightarrow \infty$. 
Thus, the unrestricted Hartree-Fock orbitals based on asymptotic projection lead to wave function and energy expressions which are equivalent to those obtained by the Roothaan method. However, the asymptotic projection approach has some advantages:

- The method avoids introducing the off-diagonal Lagrange multipliers coupling the closed-shell and open-shell orbitals and, therefore, arbitrariness in the definition of the Fock operators does not arise. Equations (25) and (26) are cubic equations with respect to orbital expansion coefficients (lcao) and can be easily implemented in established unrestricted Hartree-Fock codes. The 'traditional' open-shell Roothaan-based methods give rise to equations of fifth and seventh degree with respect to these coefficients.

- The scheme defined in Eqs. (25) and (26) provides a well-defined zero-order approximation for open-shell many-body perturbation theory that ensures that single excitations do not contribute to the second-order energy. This should be contrasted with the method of Knowles et al. [21], the restricted MøllerPlesset approach, and the method of Amos et al. [20], here designated AAHK, which employ different orbitals for different spins and for which the generalized Brillouin's theorem is not satisfied and consequently single replacement contributions enter the second-order energy expression.

The structure of the Fock matrices occurring in the asymptotic projection-selfconsistent field method is displayed in Fig. 1. The structure of the Fock matrices arising in the RMP and the AAHK methods is shown in Figs. 2 and 3, respectively.

The asymptotic projection-self-consistent field equations are similar to those reported for the RMP and AAHK methods. However, it should be emphasized that Eqs. (25) and (26) give rise to spatial parts of the $\alpha$ set which are identical to those of $\beta$ set. In contrast, for the RMP and AAHK methods the $\alpha$ orbitals are not coincident with the $\beta$ orbitals [21] since these orbitals are rotated with respect to the orbitals given by the Roothaan procedure and Brillouin's theorem is not obeyed. The differences between the methods is evident when we compare the Fock matrices $F^{\alpha}$ and $F^{\beta}$. It can be seen in Fig. (1) that $s+v$ block of the asymptotic projection-selfconsistent field $F^{\alpha}$ matrix has diagonal form and, therefore, the condition (31) is satisfied. Furthermore, the diagonal form of $d+s$ block of the $F^{\beta}$ matrix confirms that condition (30) is also satisfied. However, it is clear by inspection of Figs. (2) and (3) for the RMP and AAHK approaches that these do not satisfy Brillouin's theorem.

\subsubsection{Matrix Hartree-Fock Energies for Ground States}

In this subsection, some features of the alternative self-consistent field procedure based on the asymptotic projection technique are demonstrated by means of HartreeFock calculations of the ground state energies for the $\mathrm{HeH}$ and $\mathrm{BeH}$ molecules. The Hartree-Fock ground state for the three-electron $\mathrm{HeH}$ molecule with electronic configuration $1 \sigma^{2} 2 \sigma$ is unbound. In this study, we used a $\mathrm{He}-\mathrm{H}$ distance of $1.500 \mathrm{bohr}$, 
Fig. 1 Structure of the Fock matrices in the asymptotic projection method. $d$ denotes the doubly occupied component of the orbital space, $s$ denotes the singly occupied component and $v$ the virtual (or unoccupied) part. denotes a non-zero element of the matrix
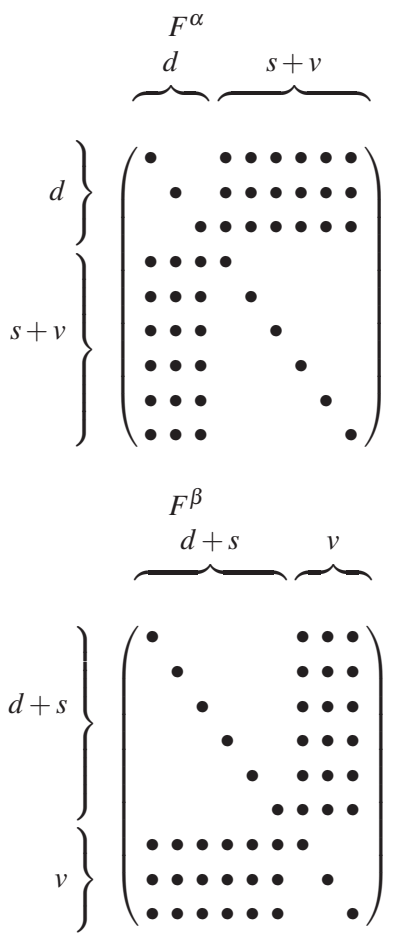

which corresponds to the minimum in the potential energy curve for the first excited $\Sigma$ state. To assess the potential of the approach based on asymptotic projection, we compare the matrix Hartree-Fock energies with the finite difference ROHF energy obtained on a grid designated [217 $\times 349 ; 65]$ (J. Kobus, 2004, personal communication): $E_{\mathrm{fdHF}}=-3.220315124616$ hartree which provides an exact Hartree-Fock result to near-machine accuracy.

The ground state of the five-electron $\mathrm{BeH}$ molecule with the electronic configuration $1 \sigma^{2} 2 \sigma^{2} 3 \sigma$ was studied for a nuclear separation of $2.500 \mathrm{bohr}$. The finite difference ROHF energy of this molecule at this geometry is $E_{\mathrm{fdHF}}=-15.15318233996$ hartree (J. Kobus, 2004, personal communication).

Self-consistent field energy calculations were carried out using a single reference wave function. For all calculations, basis sets of $s$-type Gaussian functions were used with exponents and positions determined by minimizing the Hartree-Fock energy. Equation (27) were used to construct numerical procedures for basis set optimizations. It has been found recently that such basis sets are capable of supporting an accuracy at the sub- $\mu$ hartree level for simple molecules $[42,44]$. The expectation value of the energy given by the alternative SCF method depends on a parameter $\lambda$, where $\lambda=0$ corresponds to the UHF energy and tends asymptotically to the ROHF energy given by Roothaan's method as $\lambda \rightarrow \infty$. Table 1 presents the calculated energies for $\mathrm{HeH}$ as a function of $\lambda$ together with the expectation value $\left\langle S^{2}\right\rangle$ for the case of $14 s$ basis functions. We can see that setting $\lambda=100000$ yields practically 
Fig. 2 Structure of the Fock matrices in the restricted Møller-Plesset method. $d$ denotes the doubly occupied component of the orbital space, $s$ denotes the singly occupied component and $v$ the virtual (or unoccupied) part. • denotes a non-zero element of the matrix

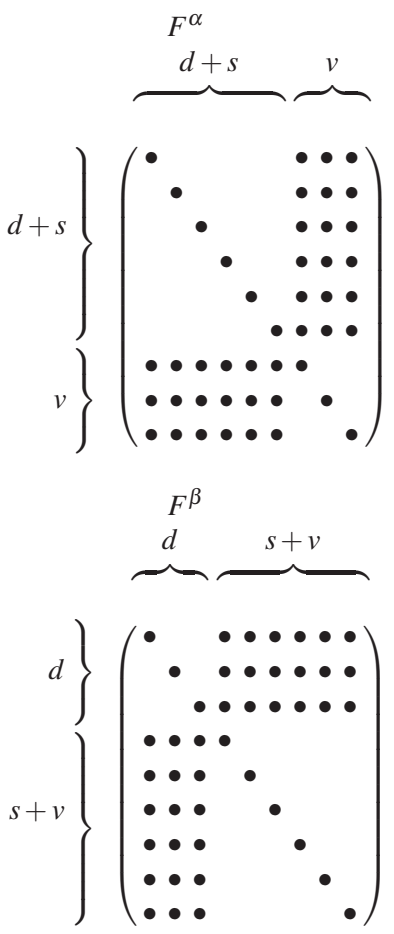

the energy obtained by Roothaan's method ( $E_{\text {Roothaan }}=-3.21919891$ hartree). For $\lambda \sim 5,000-10,000$, we achieve a sub- $\mu$ hartree level of agreement between our method and the Roothaan method. The expectation value $\left\langle S^{2}\right\rangle$ is given to our target accuracy for values $\lambda>\sim 100-500$. In Table 2 , we record the total restricted open-shell matrix Hartree-Fock energies for Gaussian s-type basis sets of increasing size. In this table, $N$ denotes the total number of basis functions in a given basis set. $\Delta$ is the difference, in $\mu$ hartree, between successive entries in Table $2 . \Delta_{n}$ is the value of $\Delta$ divided by the number of basis functions added between successive rows of Table 2. $\delta$ is the difference, again in $\mu$ hartree, between the matrix HartreeFock energy supported by a given basis set and corresponding value obtained by the finite difference method. It can be seen that the largest basis set considered in Table 2, which contains $48 s$-type Gaussian functions, supports an accuracy at the sub- $\mu$ hartree level. The energy reported for this basis set corresponds to $\lambda=6,000$.

Table 3 illustrates the dependence of the open-shell matrix Hartree-Fock energy and $\left\langle S^{2}\right\rangle$ for the $\mathrm{BeH}$ molecule on the parameter $\lambda$. The basis set of 30 s-type Gaussian functions was used in this case. Values of $\lambda$ in the range $\sim 5,000-10,000$ provided the required accuracy in the energy when compared with the traditional Roothaan method. We can see that the study of the $\mathrm{BeH}$ molecule is similar to that of $\mathrm{HeH}$ molecule, in particular, it can be seen that when $\lambda$ increases the expectation value of the square of the spin operator $\left\langle S^{2}\right\rangle$ decreases much more rapidly than the total energy increases. The total restricted open-shell Hartree-Fock energies are 
Fig. 3 Structure of the Fock matrices in the method of Amos, Andrews, Handy and Knowles (AAHK). $d$ denotes the doubly occupied component of the orbital space, $s$ denotes the singly occupied component and $v$ the virtual (or unoccupied) part. • denotes a non-zero element of the matrix

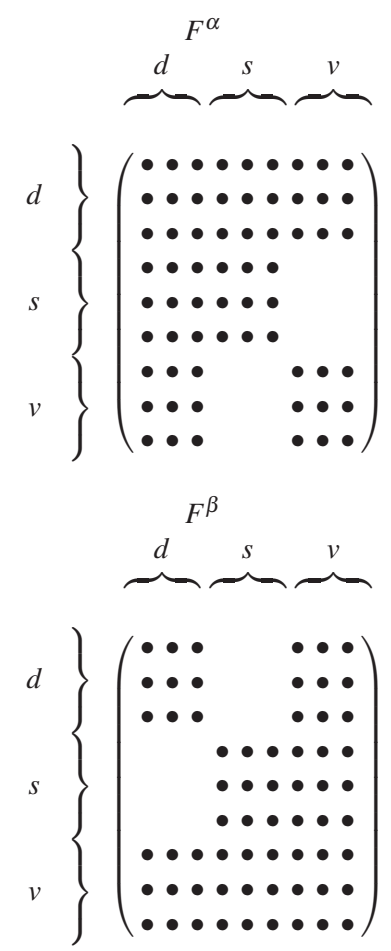

Table 1 Dependence of the open-shell matrix Hartree-Fock energy based on asymptotic projection on the parameter $\lambda$ for the ground state of the $\mathrm{HeH}$ molecule at $R=1.5$ bohr with the basis set of $14 s$ functions. Atomic units are used

\begin{tabular}{ccc}
\hline$\lambda^{\mathrm{a}}$ & $\left\langle S^{2}\right\rangle$ & Energy \\
\hline 0 & 0.75042893 & -3.21976561 \\
50 & 0.75000031 & -3.21930832 \\
100 & 0.75000008 & -3.21921527 \\
500 & 0.75000000 & -3.21920225 \\
5,000 & 0.75000000 & -3.21919924 \\
10,000 & 0.75000000 & -3.21919907 \\
100,000 & 0.75000000 & -3.21919892 \\
\hline
\end{tabular}

a The value $\lambda=0$ corresponds to the unrestricted Hartree-Fock energy, whereas $\lambda=100,000$ yields practically the energy obtained by the Roothaan method $\left(E_{\text {Roothaan }}=-3.21919891\right.$ hartree).

given in Table 4 for basis sets of increasing size. The largest basis set considered in this table contains 62 functions and supports an accuracy at the sub- $\mu$ hartree level. A total energy of -15.15318143 hartree was obtained with $\lambda=10,000$ a.u. which lies $0.91 \mu$ hartree above the finite difference Hartree-Fock energy.

In concluding this section, we point out that optimal basis set parameters for the largest basis sets for the $\mathrm{HeH}$ and $\mathrm{BeH}$ molecules can be found in Ref. [44]. 
Table 2 Convergence of the open-shell matrix Hartree-Fock-asymptotic projection energy for the ground state of the $\mathrm{HeH}$ molecule, at $R=1.5 \mathrm{bohr}$, as a function of the basis set size. Atomic units are used

\begin{tabular}{ccccc}
\hline$N$ & Energy $^{\mathrm{a}}$ & $\Delta$ & $\Delta_{n}$ & $\delta$ \\
\hline 14 & -3.21919907 & - & - & 1116.05 \\
24 & -3.21920172 & 1002.65 & 100.27 & 113.40 \\
30 & -3.21929493 & 93.214 & 15.54 & 20.19 \\
43 & -3.21931319 & 18.26 & 1.40 & 1.93 \\
48 & -3.21931434 & 1.15 & 0.23 & 0.78 \\
\hline
\end{tabular}

a The value $\lambda=10,000$ was used. The energy differences, $\Delta, \Delta_{n}$ and $\delta$, which are defined in the text, are given in $\mu$ hartree.

Table 3 Dependence of the open-shell matrix Hartree-Fock-asymptotic projection energy on the parameter $\lambda$ for the ground state of the $\mathrm{BeH}$ molecule at $R=2.5$ bohr with the basis set of 30 s-type Gaussian functions. Atomic units are used

\begin{tabular}{ccc}
\hline$\lambda^{\mathrm{a}}$ & $\left\langle S^{2}\right\rangle$ & Energy \\
\hline 0 & 0.75178828 & -15.15342893 \\
100 & 0.75000012 & -15.15309116 \\
1,000 & 0.75000000 & -15.15308913 \\
5,000 & 0.75000000 & -15.15308913 \\
6,000 & 0.75000000 & -15.15308892 \\
10,000 & 0.75000000 & -15.15308890
\end{tabular}

a Values of $\lambda=$ in the range $\sim 5,000-10,000$ provide the required accuracy when compared with the traditional Roothaan method.

Table 4 Convergence of the open-shell matrix Hartree-Fock-asymptotic projection energy for the ground state of the $\mathrm{BeH}$ molecule at $R=2.5 \mathrm{bohr}$, as a function of the basis set size. Atomic units are used

\begin{tabular}{ccccc}
\hline$N$ & Energy $^{\mathrm{a}}$ & $\Delta$ & $\Delta_{n}$ & $\delta$ \\
\hline 24 & -15.15245700 & - & - & 725.34 \\
30 & -15.15308892 & 631.92 & 105.35 & 93.42 \\
36 & -15.15314032 & 51.40 & 8.57 & 42.02 \\
43 & -15.15316408 & 23.76 & 3.39 & 18.26 \\
60 & -15.15317941 & 15.33 & 0.90 & 2.93 \\
62 & -15.15318143 & 2.02 & 0.01 & 0.91
\end{tabular}

a The value $\lambda=10,000$ was used. The energy differences, $\Delta, \Delta_{n}$ and $\delta$, which are defined in the text, are given in $\mu$ hartree.

\section{Excited State SCF Theory Based on the Asymptotic Projection Method}

\subsection{Specific Features of Excited State SCF Calculations}

Existing open-shell self-consistent field methods for ground states cannot be applied directly to excited states of the same symmetry as a lower state without "variational 
collapse'; that is, the approximation to the excited state wave function is contaminated by components of a lower state. Several useful methods have been proposed to overcome the 'variational collapse' problem and a number of different schemes have been proposed for obtaining Hartree-Fock wave functions for excited states [45-50], [34,36]. Some of these approaches [45-47], [34,36,50] explicitly introduce orthogonality constraints to lower states. Other methods [48], [49] introduce this restriction implicitly. In both types of scheme, the excited state self-consistent field wave function of interest is orthogonal to the wave function for a lower state or states of the same symmetry, but this lower state or states are not necessarily the best self-consistent field functions for these states [50]. An interesting ensemble Hartree-Fock approach [51] based on the extended Raleigh-Ritz variational principle [52] have been also proposed. This is a good compromise in applications to the excited state problem within the framework of density functional theory and has found application in wave function based formulation as well [35]. In particular, calculations for atoms have showed that the ensemble Kohn-Sham theory, with the exact ensemble-exchange potential, can be as accurate as the ground state calculations [53,54]. However, partly due to the lack so far of an accurate correlation energy functional, there exist very few reported applications for molecules, where a finite basis set choice is important to achieve reasonable results. Some preliminary calculations concerning the choice of an optimal basis set for an ensemble of states can be found in the work of Glushkov and Theophilou [55,56].

\subsection{Orthogonality Constraints for Single Determinantal Wave Functions}

Before deriving the Hartree-Fock equations for the excited state orbitals, we shall consider the orthogonality constraints imposed on these orbitals.

The exact many-electron wave function for an excited state, $\Psi_{i}, i \neq 0$, satisfies orthogonality conditions with respect to other many-electron states including the ground state, $\Psi_{0}$. For example, for the first excited state with many-electron wave function $\Psi_{1}$ we have

$$
\left\langle\Psi_{0} \mid \Psi_{1}\right\rangle=0 .
$$

The exact ground state wave function, $\Psi_{0}$, can be written

$$
\Psi_{0}=\Phi_{0}+\chi_{0}
$$

where $\Phi_{0}$ is the many-electron ground state Hartree-Fock wave function and $\chi_{0}$ is the correlation correction. Without loss of generality, we can require

$$
\left\langle\Phi_{0} \mid \chi_{0}\right\rangle=0 .
$$

Similarly, the exact excited state wave function, $\Psi_{1}$, can be written

$$
\Psi_{1}=\Phi_{1}+\chi_{1}
$$


where $\Phi_{1}$ is the many-electron excited state Hartree-Fock wave function and $\chi_{1}$ is the corresponding correlation correction. Again, without loss of generality, we can require

$$
\left\langle\Phi_{1} \mid \chi_{1}\right\rangle=0 .
$$

Substituting Eq. (37) into Eq. (34) we get

$$
\begin{aligned}
\left\langle\Psi_{0} \mid \Psi_{1}\right\rangle & =\left\langle\Psi_{0} \mid \Phi_{1}\right\rangle+\left\langle\Psi_{0} \mid \chi_{1}\right\rangle \\
& =0 .
\end{aligned}
$$

If we require that

$$
\left\langle\Psi_{0} \mid \Phi_{1}\right\rangle=0
$$

which implies that

$$
\left\langle\Psi_{0} \mid \chi_{1}\right\rangle=0
$$

then it is easily shown that

$$
\frac{\left\langle\Phi_{1}|H| \Phi_{1}\right\rangle}{\left\langle\Phi_{1} \mid \Phi_{1}\right\rangle} \geqslant \mathscr{E}_{1}
$$

where $\mathscr{E}_{1}$ is the exact energy of the excited state and $H$ is the total electronic hamiltonian operator. However, Eqs. (40) and (41) cannot be used directly because the exact wave function for the ground state, $\Psi_{0}$, is unknown.

Substituting Eqs. (35) and (37) into Eq. (34) we have

$$
\begin{aligned}
\left\langle\Psi_{0} \mid \Psi_{1}\right\rangle & =\left\langle\Phi_{0} \mid \Phi_{1}\right\rangle+\left\langle\Phi_{0} \mid \chi_{1}\right\rangle+\left\langle\chi_{0} \mid \Phi_{1}\right\rangle+\left\langle\chi_{0} \mid \chi_{1}\right\rangle \\
& =0
\end{aligned}
$$

or

$$
\left\langle\Phi_{0} \mid \Phi_{1}\right\rangle=-\left[\left\langle\Phi_{0} \mid \chi_{1}\right\rangle+\left\langle\chi_{0} \mid \Phi_{1}\right\rangle+\left\langle\chi_{0} \mid \chi_{1}\right\rangle\right] .
$$

We see that the Hartree-Fock wave functions, do not, in general, satisfy orthogonality constraints analogous to those obeyed by the exact wave functions. However, we may impose constraints upon the Hartree-Fock function so that, for example,

$$
\left\langle\Phi_{0} \mid \Phi_{1}\right\rangle=0
$$

From (44) we see that this constraint requires that

$$
\left\langle\Phi_{0} \mid \chi_{1}\right\rangle+\left\langle\chi_{0} \mid \Phi_{1}\right\rangle=-\left\langle\chi_{0} \mid \chi_{1}\right\rangle
$$

The imposition of the constraint (45) on an approximate lower state wave function, such as the Hartree-Fock function, does not, in general, yield an excited state energy which is an upper bound to the exact excited state energy. An upper bound to the excited state energy is obtained if we impose the additional constraint

$$
\left\langle\Phi_{0}|H| \Phi_{1}\right\rangle=0 \text {. }
$$

In practice, if the lower state energy and the corresponding wave function are known accurately then the coupling matrix element $\left\langle\Phi_{0}|H| \Phi_{1}\right\rangle$ is small (see Part I of this 
work for more detailed discussion [27]). Experience shows that, because the finite basis set approximation is usually more restrictive for $\Phi_{1}$ than it is for $\Phi_{0}$, the calculated excited state energy lies above the corresponding exact value.

The imposition of the constraint (45) is important since

(i) any lack of orthogonality of the Hartree-Fock wave functions may lead to excited state energies lying below the corresponding exact energies. (For example, Cohen and Kelly [57] found for the He atom the first singlet excited state energy $E_{1}=-2.16984$ hartree, whereas the observed energy $E_{1, \text { exact }}=$ -2.14598 hartree. (see also the work of Tatewaki et al. [58]).);

(ii) it facilitates the development of a simple perturbation theory expansion for correlation effects in excited states [37];

(iii) it facilitates the study of properties which depend on the wave functions of different states, for example the evaluation of transition properties (see also the work of Colle et al. [50]).

We shall be concerned with ground and excited electronic states which can be adequately described by a single determinantal wave function, i.e. doublet states, triplet states, etc. with spin $S \neq 0$ ).

Let $\Phi_{0}$ be the ground state Slater determinant constructed from a set of spinorbitals consisting of spatial part $\left|\varphi_{0 i}^{\alpha}\right\rangle,\left(i^{\alpha}=1,2, \ldots, n^{\alpha}\right)$ associated with $\alpha$ spin functions and orbitals $\left|\varphi_{0 i}^{\beta}\right\rangle,\left(i^{\beta}=1,2, \ldots, n^{\beta}\right)$ associated with $\beta$ spin functions, i.e.

$$
\Phi_{0}=(N !)^{-\frac{1}{2}} \operatorname{det}\left|\varphi_{01}^{\alpha} \alpha, \ldots, \varphi_{0 n}^{\alpha} \alpha ; \varphi_{01}^{\beta} \beta, \ldots, \varphi_{0 n}^{\beta} \beta\right| .
$$

Without loss of generality, we define $n^{\alpha}>n^{\beta}, n^{\alpha}+n^{\beta}=N$, where $N$ is a number of electrons and $S=S_{z}=\left(n^{\alpha}-n^{\beta}\right) / 2$ is the total spin. Similarly, $\Phi_{1}$ is a single determinant wave function for the first excited state:

$$
\Phi_{1}=(N !)^{-\frac{1}{2}} \operatorname{det}\left|\varphi_{11}^{\alpha} \alpha, \ldots, \varphi_{1 n}^{\alpha} \alpha ; \varphi_{11}^{\beta} \beta, \ldots, \varphi_{1 n}^{\beta} \beta\right| .
$$

It is well known that the orthogonality constraint for functions (46) and (47)

$$
\left\langle\Phi_{0} \mid \Phi_{1}\right\rangle=0
$$

can be written in terms of the spatial orbitals in the form

$$
\begin{aligned}
\left\langle\Phi_{0} \mid \Phi_{1}\right\rangle= & \operatorname{det}\left|\left\langle\varphi_{01}^{\alpha} \mid \varphi_{11}^{\alpha}\right\rangle \ldots\left\langle\varphi_{0 n}^{\alpha} \mid \varphi_{1 n}^{\alpha}\right\rangle\right| \times \\
& \operatorname{det}\left|\left\langle\varphi_{01}^{\beta} \mid \varphi_{1 n}^{\alpha}\right\rangle \ldots\left\langle\varphi_{0 n}^{\beta} \mid \varphi_{1 n}^{\beta}\right\rangle\right| \\
= & 0 .
\end{aligned}
$$

The annihilation of either one of the two determinants in (49) leads to fulfillment of the orthogonality condition (48). From energy considerations and previous computational experience, we impose the orthogonality restrictions only via the first 
determinant which is associated with the $\alpha$ set and involves the occupied orbital highest in energy.

As is well known, the condition

$$
\operatorname{det}\left|\left\langle\varphi_{01}^{\alpha} \mid \varphi_{11}^{\alpha}\right\rangle \ldots\left\langle\varphi_{0 n}^{\alpha} \mid \varphi_{1 n}^{\alpha}\right\rangle\right|=0
$$

is fulfilled if either the rows or columns in the first overlap determinant are linearly dependent. Therefore, two physically different schemes are possible to satisfy (49): either

$$
\sum_{j}^{n^{\alpha}} b_{j}^{1}\left\langle\varphi_{0 i}^{\alpha} \mid \varphi_{1 j}^{\alpha}\right\rangle=0, \quad i=1,2, \ldots, n^{\alpha}
$$

or

$$
\sum_{i}^{n^{\alpha}} b_{i}^{0}\left\langle\varphi_{0 i}^{\alpha} \mid \varphi_{1 j}^{\alpha}\right\rangle=0, \quad j=1,2, \ldots, n^{\alpha} .
$$

Equation (50) requires that all occupied ground state orbitals be orthogonal to a linear combination of the excited state orbitals $\sum_{j}^{n^{\alpha}} b_{j}^{1}\left|\varphi_{1 j^{\alpha}}\right\rangle$, which describes an excited electronic state. Equation (51) requires the orthogonality of all occupied excited state orbital associated with $\alpha$ spin functions to the arbitrary vector $\sum_{i}^{n^{\alpha}} b_{i}^{0}\left|\varphi_{0 i}^{\alpha}\right\rangle$ from the subspace of the occupied ground state orbitals associated with $\alpha$ spin functions. In general, the coefficients $b_{i}^{0}$ can be determined by minimizing the excited state Hartree-Fock energy. However, calculations show that the choice

$$
\sum_{i}^{n^{\alpha}} b_{i}^{0}\left|\varphi_{0 i}^{\alpha}\right\rangle=\left|\varphi_{0 n}^{\alpha}\right\rangle
$$

where $\varphi_{0 n}^{\alpha}$ is the orbital from the ground state Slater determinant with the highest energy, leads to a minimum energy for the excited state. In the limit of a complete basis set or a common basis set for the ground and excited state the schemes defined by (50) and (51), yield the same energy values.

In this work, we use the second scheme to impose the orthogonality constraint (48), i.e. that defined by Eq. (51), which upon using (52) becomes

$$
\left\langle\varphi_{0 n}^{\alpha} \mid \varphi_{1 j}^{\alpha}\right\rangle=0, j=1,2, \ldots, n^{\alpha} .
$$

Equation (53) can be rewritten in symmetrical form, which is useful when deriving the Hartree-Fock equations, as follows:

$$
\left\langle\varphi_{1 j}^{\alpha} \mid \varphi_{0 n}^{\alpha}\right\rangle\left\langle\varphi_{0 n}^{\alpha} \mid \varphi_{1 j}^{\alpha}\right\rangle=0, j=1,2, \ldots, n^{\alpha}
$$

or, since the left-hand side of Eq. (54) is not negative

$$
\sum_{j}^{n^{\alpha}}\left\langle\varphi_{1 j}^{\alpha}\left|P_{n}^{\alpha}\right| \varphi_{1 j}^{\alpha}\right\rangle=0
$$

where $P_{n}^{\alpha}$ is the projection operator

$$
P_{n}^{\alpha}=\left|\varphi_{0 n}^{\alpha}\right\rangle\left\langle\varphi_{0 n}^{\alpha}\right|
$$




\subsection{Variational Derivation of Hartree-Fock Equations for Excited States}

We shall follow the unrestricted Hartree-Fock formalism for obtaining the ROHF functions developed in Sect. 2.2 to derive the Hartree-Fock equations for excited states. For the sake of simplicity, we restrict our attention to the first excited state. This means that we start with the minimization of the total energy expressed in terms of the UHF orbitals:

$$
\begin{aligned}
E_{1}^{U H F}= & \sum_{i}^{n^{\alpha}}\left\langle\varphi_{1 i}^{\alpha}|h| \varphi_{1 i}^{\alpha}\right\rangle+\frac{1}{2} \sum_{i, j}^{n^{\alpha}}\left[\left(\varphi_{1 i}^{\alpha} \varphi_{1 i}^{\alpha} \mid \varphi_{1 j}^{\alpha} \varphi_{1 j}^{\alpha}\right)-\left(\varphi_{1 i}^{\alpha} \varphi_{1 j}^{\alpha} \mid \varphi_{1 i}^{\alpha} \varphi_{1 j}^{\alpha}\right)\right] \\
& +\sum_{i}^{n^{\beta}}\left\langle\varphi_{1 i}^{\beta}|h| \varphi_{1 i}^{\beta}\right\rangle+\frac{1}{2} \sum_{i, j}^{n^{\beta}}\left[\left(\varphi_{1 i}^{\beta} \varphi_{1 i}^{\beta} \mid \varphi_{1 j}^{\beta} \varphi_{1 j}^{\beta}\right)-\left(\varphi_{1 i}^{\beta} \varphi_{1 j}^{\beta} \mid \varphi_{1 i}^{\beta} \varphi_{1 j}^{\beta}\right)\right] \\
& +\sum_{i}^{n^{\alpha}} \sum_{i}^{n^{\beta}}\left(\varphi_{1 i}^{\alpha} \varphi_{1 i}^{\alpha} \mid \varphi_{1 j}^{\beta} \varphi_{1 j}^{\beta}\right)
\end{aligned}
$$

subject to the following constraints:

(i) orbitals must satisfy the restrictions (55) which ensure the orthogonality of Slater determinants for the ground state and the first excited state;

(ii) the excited Slater determinant must be an eigenfunction of the $\mathbf{S}^{2}$ operator, i.e. we impose the spin purity condition in the form

$$
\sum_{k}^{n^{\beta}}\left\langle\varphi_{1 k}^{\beta}\left|Q^{\alpha}\right| \varphi_{1 k}^{\beta}\right\rangle=0
$$

where $Q^{\alpha}=I-P^{\alpha}$ is the orthoprojector on the subspace of the virtual $\alpha$ spin orbitals. It is useful to remember that Eq. (57) implies that the set of orbitals associated with the $\beta$ spin functions lies completely within the space defined by the set associated with the $\alpha$ spin functions.

The Hartree-Fock equations for the excited state orbitals can now be obtained by constructing a functional consisting of the UHF energy expression together with terms imposing the orthogonality constraints (55) and (57) by the method of Lagrange undetermined multipliers. In particular, the constraints (55) and (57) multiplied by Lagrange multipliers $\lambda_{1}$ and $\lambda_{2}$, respectively, are added to the UHF energy $E_{1}^{U H F}=\left\langle\Phi_{1}|H| \mid \Phi_{1}\right\rangle$, so as to give the following functional

$$
L=E_{1}^{U H F}+\lambda_{1} \sum_{i}^{n^{\beta}}\left\langle\varphi_{1 i}^{\beta}\left|Q^{\alpha}\right| \varphi_{1 i}^{\beta}\right\rangle+\lambda_{2} \sum_{i}^{n^{\alpha}}\left\langle\varphi_{1 j}^{\alpha}\left|P_{n}^{\alpha}\right| \varphi_{1 j}^{\alpha}\right\rangle .
$$


The stationary condition has the form

$$
\delta L=\delta\left[E_{1}^{U H F}+\lambda_{1} \sum_{i}^{n^{\beta}}\left\langle\varphi_{1 i}^{\beta}\left|Q^{\alpha}\right| \varphi_{1 i}^{\beta}\right\rangle+\lambda_{2} \sum_{i}^{n^{\alpha}}\left\langle\varphi_{1 j}^{\alpha}\left|P_{n}^{\alpha}\right| \varphi_{1 j}^{\alpha}\right\rangle\right]=0 .
$$

It should be emphasised that, although this condition is based on the UHF energy expression, it leads to a result corresponding to ROHF theory.

In practical applications, we invariably invoke the algebraic approximation by parameterizing the orbitals in a finite basis set. This approximation may be written

$$
\left|\varphi_{1}\right\rangle=P\left|\varphi_{1}\right\rangle
$$

where $P$ is an orthoprojector defined by a chosen basis set with dimension $M_{1}$, i.e.

$$
P=\sum_{p, q}^{M_{1}}\left|\chi_{p}^{1}\right\rangle\left(\mathbf{S}^{-1}\right)_{p q}\left\langle\chi_{q}^{1}\right|
$$

where $\mathbf{S}$ is the overlap matrix and $\mathbf{S}^{-1}$ is its inverse.

It should be stressed that, in general, the basis set for the excited state,

$$
\left\{\chi_{p}^{1} ; p=1,2, \ldots, M_{1}\right\},
$$

is distinct from that for the ground state,

$$
\left\{\chi_{p}^{0} ; p=1,2, \ldots, M_{0}\right\} .
$$

The stationary condition (59) leads, after some manipulation, to the following equations

$$
\begin{aligned}
& \lim _{\lambda_{1}, \lambda_{2} \rightarrow \infty} P\left(\mathbf{F}^{\alpha}-\lambda_{1} P^{\beta}+\lambda_{2} P_{n}^{\alpha}-\varepsilon_{i}^{a}\right) P\left|\varphi_{1 i}^{\alpha}\right\rangle=0, i^{\alpha}=1,2, \ldots, n^{\alpha}, \ldots, M_{1} \\
& \lim _{\lambda_{1} \rightarrow \infty} P\left(\mathbf{F}^{\beta}+\lambda_{1} Q^{\alpha}-\varepsilon_{i}^{\beta}\right) P\left|\varphi_{1 i}^{\beta}\right\rangle=0,
\end{aligned}
$$

Here $\mathbf{F}^{\alpha}$ and $\mathbf{F}^{\beta}$ are the standard UHF operators constructed from the excited state orbitals $\varphi_{1 i}$. According to the asymptotic projection technique the terms $\lambda_{1} P^{\beta}$ and $\lambda_{1} Q^{\alpha}, \lambda_{1} \rightarrow \infty$, ensure spin purity (see the work of Glushkov and Tsaune [34] and also Glushkov [24] for more details) whereas the term $\lambda_{2} P_{n}^{\alpha}, \lambda_{2} \rightarrow \infty$, provides the orthogonality of states.

This result can be easily extended to the higher energy levels. For example, for the second excited state the operator $P_{n}^{\alpha}$ should be substituted by the orthoprojector

$$
P_{n}^{\alpha}=\left|\varphi_{0 n}^{\alpha}\right\rangle\left\langle\varphi_{0 n}^{\alpha}|+| \varphi_{1 n}^{\alpha}\right\rangle\left\langle\varphi_{1 n}^{\alpha}\right|
$$

etc., i.e. the problem of choosing a determinantal wave function for the higher excitations does not arise. The only additional computation beyond that required for the UHF scheme is the evaluation of the overlap matrix element $\left\langle\varphi_{0 n}^{\alpha} \mid \varphi_{1 j}^{\alpha}\right\rangle$. It should 
be noted that for practical calculations, the value $\lambda_{1} \sim 100-500$ a.u. ensures spin purity [24], whereas $\lambda_{2} \sim 1,000$ a.u. ensures that $\left\langle\Phi_{0} \mid \Phi_{1}\right\rangle<10^{-6}-10^{-7}$ [36].

It is worth also noting that because of the asymptotic projection method, all excited configurations based on the excited Slater determinant $\Phi_{1}$, viz., $\Phi_{1 i}^{a}, \Phi_{1 i j}^{a b}$, etc., are orthogonal both to $\Phi_{0}$ and among themselves. Therefore, these functions form the orthonormal basis set in the many-body space and can be used, unlike other SCF methods which do not satisfy the orthogonality of states in the explicit form, to develop many-body methods incorporating the correlation effects, in particular, a many-body Møller-Plesset-like perturbation theory (see Sect. 5).

\subsection{Numerical Results and Discussion}

At present, there are only very few published finite basis set calculations for excited states having the same symmetry as the ground state which are based on existing Hartree-Fock methods. For some atoms, numerical Hartree-Fock (NHF) results are available [59]. They can be used to examine the performance of the excited state SCF theory presented above.

\subsubsection{Even-Tempered Basis Set Implementation for Excited States of Atoms}

In this section, we describe three different schemes for developing systematic sequences of even-tempered basis sets for excited states. We define each scheme, which we label $(a),(b)$ and $(c)$ in turn.

In each scheme, we generate a sequence of even-tempered basis sets, with exponents given by

$$
\zeta_{p}=\alpha \beta^{p}, p=1,2, \ldots, M .
$$

Following Schmidt and Rudenberg(61), in the following we shall use $\alpha$ and $\beta$ for the parameters that generate a sequence of even-tempered basis sets and not for spin function as we did above. The parameters $\alpha$ and $\beta$ must be taken to be functions of $M$, the number of basis functions, i.e. $\alpha=\alpha(M)$ and $\beta=\beta(M)$, if the Gaussian sets defined by (61) are to become complete in the appropriate subspace as $M \rightarrow \infty$.

In the scheme which we label $(a)$, the same basis set is employed for both the ground and excited state. Therefore, the same integrals over basis functions are used for both states. The values of the even-tempered parameters $\alpha$ and $\beta$ are those which were optimized for the ground state of the atom as reported by Schmidt and Ruedenberg [60]. These values are given in Table 1 of Schimdt and Ruedenberg's paper [60].

In scheme $(b)$ the basis set is optimized by invoking the variation principle for each state considered. For the ground state the optimized values of the even-tempered parameters $\alpha$ and $\beta$ given by Schmidt and Ruedenberg [60] are used whereas for the excited state optimal values for a sequence of $M$ values are determined by minimizing the corresponding excited state energy. 
In our third scheme, which we label $(c)$, we optimized the parameter $\alpha$ and $\beta$ for the smallest basis set considered, i.e. $M=6$ and then determine values of these parameters for the basis sets of larger size by using the recursions [61]:

$$
\alpha[M]=\left[\frac{\beta[M]-1}{\beta[M-1]-1}\right]^{a} \alpha[M-1]
$$

and

$$
\ln (\beta[M])=\left[\frac{M}{M-1}\right]^{b} \ln \beta[M-1]
$$

with the values of $a$ and $b$ taken from the work of Schmidt and Ruedenberg [60]. These values are given in Table 3 of reference [60].

We performed prototype calculations on some simple atoms in order to study the rate of convergence of the excited state energies and the accuracy which could be supported before problems associated with the precision of our calculations arising from computational linear dependence became significant. In particular, we studied the ${ }^{3} S$ states of the He atom corresponding to the configurations $1 s 2 s, 1 s 3 s$ and $1 s 4 s$, and the ${ }^{3} S$ states of the Be atom corresponding to the configurations ( $\left.\mathrm{He}\right) 2 s 3 s$ and (He) $2 s 4 s$.

Using scheme $(b)$ we also computed excitation energies for the Be isoelectronic series in a comparison with the numerical Hartree-Fock values.

\subsubsection{Matrix Hartree-Fock Energies and Excitation Energies of Atoms}

The ground and excited state matrix Hartree-Fock energies for the $\mathrm{He}$ and $\mathrm{Be}$ atoms are presented in Tables 5 and 6, respectively. All energies are given in atomic units, (Hartree). In each of these tables, we label the columns according to the three schemes, $(a),(b)$ and $(c)$, described above. We consider each system in turn.

For the excited states of the He atom considered in Table 8 the numerical HartreeFock energies are known from the work of Froese-Fischer [59] to be $-E(1 s 2 s)=$ -2.17426 Hartree, $E(1 s 3 s)=-2.06849$ Hartree, $E(1 s 4 s)=-2.03644$ Hartree. For none of the three states considered does the sequence of basis sets constructed according to scheme $(a)$ achieve satisfactory accuracy. For the $1 s 2 s$ state, the energy supported by the largest basis set, i.e. $M=72$, is in error by $\sim 0.8 m$ Hartree. For the $1 s 3 s$ state this error is $\sim 0.056$ Hartree, whilst for the $1 s 4 s$ state the calculation with the largest basis set failed to converge and for the next largest set the error is $\sim 0.348$ Hartree. Not surprisingly, a basis set designed for the ground state supports an increasingly poor description of excited states as the level of excitation increases. Equally, it is not surprising that if the sequence of even-tempered basis sets for each excited state is optimized independently then the matrix Hartree-Fock energies converge to values in good agreement with the corresponding numerical HartreeFock energies. What is more this level of agreement is achieved for basis sets of only 42 functions in the case of the He states considered here. Scheme $(c)$ leads to sequences of energies which begin, of course, with values equal to those for scheme 
Table 5 Self-Consistent Field energies (in hartree) of the He atom for some ${ }^{3} \mathrm{~S}$ excited states as a function of the size, $M$, of the even-tempered basis set used to parameterize the orbitals. In the column headed $(a)$ : the same even-tempered basis set - optimized for the ground state, is used for all states; $(b)$ : the even-tempered basis set is optimized for each state; $(c)$ : the even-tempered parameters $\alpha$ and $\beta$ are optimized for each basis set for the smallest basis set $(M=6)$ and larger basis sets are generated using the recursions

\begin{tabular}{|c|c|c|c|}
\hline \multirow[t]{2}{*}{$M$} & \multicolumn{3}{|c|}{${ }^{3} \mathrm{~S} 1 s 2 s$} \\
\hline & (a) & (b) & (c) \\
\hline 6 & -1.83461514 & -2.16969148 & -2.16969148 \\
\hline 12 & -2.09589565 & -2.17420691 & -2.17416233 \\
\hline 18 & -2.14125461 & -2.17424990 & -2.17424758 \\
\hline 24 & -2.15704132 & -2.17425075 & -2.17425059 \\
\hline 30 & -2.16421386 & -2.17425077 & -2.17425076 \\
\hline 42 & -2.17012977 & -2.17425078 & -2.17425078 \\
\hline 54 & -2.17228698 & - & - \\
\hline 60 & -2.17284371 & - & - \\
\hline 72 & -2.17348174 & - & - \\
\hline \multirow[t]{2}{*}{$M$} & \multicolumn{3}{|c|}{${ }^{3} \mathrm{~S} 1 s 3 s$} \\
\hline & (a) & (b) & (c) \\
\hline 6 & 1.73706970 & -2.05902284 & -2.05902285 \\
\hline 12 & -1.10978995 & -2.06833906 & -2.06768910 \\
\hline 18 & -1.58429285 & -2.06847975 & -2.06845041 \\
\hline 24 & -1.76064449 & -2.06848472 & -2.06847385 \\
\hline 30 & -1.84981446 & -2.06848493 & -2.06848386 \\
\hline 42 & -1.93754688 & -2.06848496 & -2.06848488 \\
\hline 54 & -1.97977236 & - & - \\
\hline 60 & -1.99338333 & - & - \\
\hline 72 & -2.01255580 & - & - \\
\hline \multirow[t]{2}{*}{$M$} & \multicolumn{3}{|l|}{${ }^{3} \mathrm{~S} 1 s 4 s$} \\
\hline & (a) & (b) & (c) \\
\hline 6 & 17.38262804 & -2.00192936 & -2.00192936 \\
\hline 12 & 2.01602912 & -2.03624788 & -2.03501230 \\
\hline 18 & -0.09897153 & -2.03641613 & -2.03601202 \\
\hline 24 & -0.81661258 & -2.03643495 & -2.03641298 \\
\hline 30 & -1.16049508 & -2.03643584 & -2.03641853 \\
\hline 42 & -1.48612549 & -2.03643641 & -2.03643607 \\
\hline 54 & -1.64005315 & - & - \\
\hline 60 & -1.68986935 & - & - \\
\hline 72 & - & - & - \\
\hline
\end{tabular}

(b) when $M=6$ and converge almost as rapidly towards the numerical HartreeFock values. For the $1 s 2 s$ state schemes $(b)$ and $(c)$ lead to energies which agree to all figures quoted, i.e. $0.01 \mu$ Hartree when $M=42$. For the $1 s 3 s$ state there is a difference of $\sim 0.08 \mu$ Hartree between the energies supported by the two schemes when $M=42$. The corresponding difference for the $1 s 4 s$ state is $\sim 0.34 \mu$ Hartree.

For the two excited states of the Be atom considered in Table 6 the numerical Hartree-Fock energies are known [59] to be as follows: $E([\mathrm{He}] 2 s 3 s)=-14.37754$ 
Table 6 Self-Consistent Field energies (in hartree) of the Be atom for some ${ }^{3} \mathrm{~S}$ excited states as a function of the size, $M$, of the even-tempered basis set used to parameterize the orbitals. In the column headed $(a)$ : the same even-tempered basis set - optimized for the ground state, is used for all states; $(b)$ : the even-tempered basis set is optimized for each state; $(c)$ : the even-tempered parameters $\alpha$ and $\beta$ are optimized for each basis set for the smallest basis set $(M=6)$ and larger basis sets are generated using the recursions

\begin{tabular}{rccc}
\hline$M$ & \multicolumn{3}{c}{$(\mathrm{He}) 2 s 3 s$} \\
\cline { 2 - 4 } & $(a)$ & $(b)$ & $(c)$ \\
\hline 6 & -13.68597324 & -14.28743398 & -14.28743398 \\
12 & -14.30875164 & -14.37655131 & -14.37615739 \\
18 & -14.35583967 & -14.37747784 & -14.37745253 \\
24 & -14.36814873 & -14.37749790 & -14.37749650 \\
30 & -14.37282326 & -14.37749869 & -14.37749856 \\
42 & -14.37602885 & -14.37749874 & -14.37749874 \\
54 & -14.37694934 & - & - \\
60 & -14.37714828 & - & - \\
72 & -14.37735193 & - & - \\
\hline$M$ & & $(H e) 2 s 4 s$ & $(c)$ \\
\hline 6 & $-145)$ & $(b)$ & -14.10328399 \\
12 & -13.45962762 & -14.10328399 & -14.32018366 \\
18 & -14.01343862 & -14.32455109 & -14.32446205 \\
24 & -14.15980890 & -14.32460713 & -14.32458356 \\
30 & -14.22107884 & -14.32461093 & -14.32460787 \\
42 & -14.27239938 & -14.32461121 & -14.32461113 \\
54 & -14.29355025 & - & - \\
60 & -14.29978829 & - & - \\
72 & -14.30798338 & - & - \\
\hline & & & \\
\hline
\end{tabular}

Table 7 Optimized even-tempered parameters $\alpha$ and $\beta$ for ${ }^{3} \mathrm{~S}$ excited states of $\mathrm{He}$ as a function of size of basis set

\begin{tabular}{|c|c|c|c|c|c|c|}
\hline \multirow[t]{2}{*}{$M$} & \multicolumn{2}{|c|}{$1 s 2 s$} & \multicolumn{2}{|c|}{$1 s 3 s$} & \multicolumn{2}{|c|}{$1 s 4 s$} \\
\hline & $\alpha$ & $\beta$ & $\alpha$ & $\beta$ & $\alpha$ & $\beta$ \\
\hline 6 & 0.009397 & 4.210973 & 0.001684 & 4.979103 & 0.000376 & 5.420623 \\
\hline 12 & 0.009088 & 2.641628 & 0.002742 & 2.758438 & 0.000924 & 3.033467 \\
\hline 18 & 0.008243 & 2.211957 & 0.002785 & 2.202424 & 0.001226 & 2.212713 \\
\hline 24 & 0.007443 & 1.999366 & 0.001967 & 1.999755 & 0.001232 & 1.932314 \\
\hline 30 & 0.006989 & 1.897299 & 0.002043 & 1.845165 & 0.001176 & 1.794637 \\
\hline 42 & 0.005045 & 1.702089 & 0.002176 & 1.661148 & 0.001068 & 1.666500 \\
\hline
\end{tabular}

Hartree, $E([\mathrm{He}] 2 s 4 s)=-14.32466$ Hartree. For scheme $(a)$ the iterative process failed to converge for basis sets containing more than 72 functions. For the $[\mathrm{He}] 2 s 3 \mathrm{~s}$ state, the largest basis set supports an energy expectation value which is within $\sim 0.2$ mHartree of the numerical Hartree-Fock value, whilst for the $[\mathrm{He}] 2 s 4 s$ state, the corresponding difference is $\sim 16.7 \mathrm{mHartree}$. Again, a basis set designed for the ground state supports an increasingly poor description of excited states as the 
Table 8 Optimized even-tempered parameters $\alpha$ and $\beta$ for ${ }^{3} \mathrm{~S}$ excited states of Be as a function of size of basis set

\begin{tabular}{cccccc}
\hline$M$ & \multicolumn{2}{c}{$[\mathrm{He}] 2 s 3 s$} & & \multicolumn{2}{c}{$[\mathrm{He}] 2 s 4 s$} \\
\cline { 2 - 3 } \cline { 5 - 6 } & $\alpha$ & & & $\alpha$ & $\beta$ \\
\hline 6 & 0.004884 & 5.142401 & & 0.001057 & 5.809367 \\
12 & 0.004799 & 2.947601 & & 0.001459 & 3.191957 \\
18 & 0.004552 & 2.370166 & & 0.001709 & 2.424045 \\
24 & 0.004237 & 2.083730 & & 0.001736 & 2.080558 \\
30 & 0.003912 & 1.921824 & & 0.001663 & 1.899549 \\
42 & 0.003667 & 1.745016 & & 0.001537 & 1.719092 \\
\hline
\end{tabular}

Table 9 Total energies (hartree) and excitation energies (eV) for the Be isoelectronic series

\begin{tabular}{lccc}
\hline System & State & Hartree-Fock-asymptotic projection & NHF \\
\hline $\mathrm{Be}$ & $1 s^{2} 2 s 3 s$ & -14.37748 & -14.37754 \\
& $1 s^{2} 2 s 4 s$ & -14.32455 & -14.32466 \\
& $\Delta E(3 s \rightarrow 4 s)$ & 1.440 & 1.439 \\
$\mathrm{~B}^{+}$ & $1 s^{2} 2 s 3 s$ & -23.70012 & -23.70018 \\
& $1 s^{2} 2 s 4 s$ & -23.53919 & -23.53930 \\
& $\Delta E(3 s \rightarrow 4 s)$ & 4.379 & 4.378 \\
$\mathrm{C}^{2+}$ & $1 s^{2} 2 s 3 s$ & -35.38809 & -35.38826 \\
& $1 s^{2} 2 s 4 s$ & -35.06925 & -35.06943 \\
& $\Delta E(3 s \rightarrow 4 s)$ & 8.676 & 8.676 \\
$\mathrm{O}^{4+}$ & $1 s^{2} 2 s 3 s$ & -65.85221 & -65.85258 \\
& $1 s^{2} 2 s 4 s$ & -65.07035 & -65.07069 \\
& $\Delta E(3 s \rightarrow 4 s)$ & 21.276 & 21.277 \\
\hline
\end{tabular}

level of excitation increases. When the sequence of even-tempered basis sets are individually optimized for a particular state (scheme $(b)$ ) an accuracy of $\sim 0.04$ mHartree is supported for the $[\mathrm{He}] 2 s 3 s$ state and $\sim 0.05$ mHartree for the $[\mathrm{He}] 2 s 4 s$ state. A comparable accuracy is observed for the excited state energies of the $\mathrm{Be}$ atom corresponding to scheme $(c)$ in which only the basis set for $M=6$ is optimized.

The even-tempered basis set parameters obtained by optimization of $\alpha$ and $\beta$ with respect to the energy for each size of a basis set, that is scheme $(b)$ can be found in Table 7 for the He atom and in Table 8 for the beryllium atom.

Table 9 lists excitation energies (in eV) for $\mathrm{Be}, \mathrm{B}^{+}, \mathrm{C}^{2+}$ and $\mathrm{O}^{4+}$ computed with the even-tempered basis set of 18 s-type functions (scheme $(b)$ ). In Tables 9, 10 and 11 we label our implementation of SCF method the "HF - asymptotic projection" technique. The numerical Hartree-Fock energies are given in the column headed "NHF". The results of the Table 9 show that the HF - asymptotic projection method for excited states is capable of supporting high accuracy both for excited state energies and for excitation energies of the atoms and ions. 
Table 10 Total ground and some excited state energies of $\mathrm{HeH}$ at $R=1.5 \mathrm{bohr}$. Atomic units are used

\begin{tabular}{lccc}
\hline State & $\begin{array}{c}\text { Hartree-Fock - } \\
\text { asymptotic projection }\end{array}$ & $C I^{\mathrm{a}}$ & $\frac{E^{H F}-E^{C l}}{E^{C I}} \%$ \\
\hline$X^{2} \Sigma^{+}$ & -3.219851 & -3.263164 & 1.3 \\
$A^{2} \Sigma^{+}$ & -3.066606 & -3.112706 & 1.5 \\
$C^{2} \Sigma^{+}$ & -3.014785 & -3.055797 & 1.3 \\
$D^{2} \Sigma^{+}$ & -2.988232 & -3.030025 & 1.4 \\
\hline
\end{tabular}

a - Hartree-Fock energies based on the asymptotic projection method.

$\mathrm{b}$ - configuration interaction method.

Table 11 Vertical excitation energies $(\mathrm{eV})$ from $A^{2} \Sigma^{+}$of $\mathrm{HeH}$ at $R=1.5 \mathrm{bohr}$

\begin{tabular}{lccc}
\hline State & $\begin{array}{c}\text { Hartree-Fock- } \\
\text { asymptotic projection }\end{array}$ & $C I^{\mathrm{a}}$ [62] Expt [62] \\
\hline$A^{2} \Sigma^{+}$ & 0 & 0 & 0 \\
$C^{2} \Sigma^{+}$ & 1.41 & 1.53 & 1.55 \\
$D^{2} \Sigma^{+}$ & 2.13 & 2.25 & 2.26 \\
\hline
\end{tabular}

a - Hartree-Fock energies based on the asymptotic projection method.

$\mathrm{b}$ - configuration interaction method.

\subsubsection{Matrix Hartree-Fock Energies and Excitation Energies of the HeH Molecule}

The HeH molecule is of experimental interest and has been studied using accurate ab initio calculations (see, e.g. the work of Petsalakis et al. [62]), which provide excellent reference data. These data were obtained with the configuration interaction (CI) method. The CI space consisted of 4,732 configurations. Certainly, a comparison of absolute values computed by the present Hartree-Fock method with those given by the CI method would not be useful. However, the comparison of relative errors for different states is of value and permits an assessment of the performance of the method. Basis sets consisting of $18 \mathrm{~s}$ Gaussian functions were used for all states under consideration $\left(X^{2} \Sigma^{+}, A^{2} \Sigma^{+}, C^{2} \Sigma^{+}\right.$and $\left.D^{2} \Sigma^{+}\right)$. All basis functions were centred on points lying on the line passing through the nuclei (the $z$-axis). The He nucleus was placed at the origin $(0,0,0)$ and the $\mathrm{H}$ nucleus at the point $(0,0,1.500)$.

The total energy of each excited state was minimized to determine non-linear basis set parameters (orbital exponents and positions) for a given state i.e. basis sets were individually optimized for each state. By exploiting the asymptotic projection method, this procedure takes practically the same computational time for excited states as it does for the ground state. (Some details of basis set optimization for the ground state can be found in the work of Glushkov and Wilson [42-44] and 
references therein). The values $\lambda_{1}=100$ a.u. and $\lambda_{2}=10,000$ a.u. were used to solve Eq. (60).

In Tables 10 and 11, the performance of the SCF-asymptotic projection method for excited states can be observed. In these Tables, the total energies and excitation energies are given respectively make it possible to observe (see the column HartreeFock-AP).

The degree of agreement between computed and experimental values of excitation energies can be improved by taking account of electron correlation effects. It is to this problem that we turn our attention in the next section.

\section{Many-body Møller-Plesset-Like Perturbation Theory Based on Open-Shell Asymptotic Projection Orbitals}

It is well known that the choice of the zero-order Hamiltonian, $H^{(0)}$, is crucial to the success of any perturbation theory. As we have already mentioned, this choice is known to be particularly problematic for open-shell systems. On the one hand, although the theory for the construction of the ROHF wave function, which is an eigenfunction of $\mathbf{S}^{2}$, was developed by Roothaan [1] long ago, this approach does not readily lend itself to a perturbational treatment $[20,21,23,25]$. On the other hand, the UHF theory facilitates the construction of a suitable $H^{(0)}$, but the UHF Slater determinant is not, of course, an eigenfunction of $\mathbf{S}^{2}$. At present, the open-shell perturbation theory based on the so-called RMP method [21] is widely used. It is, for example, incorporated in computational quantum chemistry software packages such as GAMESS [24]. However, as we have pointed out, this method employs different orbitals for different spins for which the generalized Brillouin's theorem is not satisfied. Consequently, singly excited configurations enter the expansion for the energy at second-order. This also complicates higher-order perturbation theory calculations. In contrast, by employing an optimum set of asymptotic projection orbitals, we can develop a well-defined open-shell perturbative treatment which is a natural extension of the widely used closed-shell many-body Møller-Plesset perturbation theory. This new open-shell formalism leads to algorithms which exhibit computational costs comparable with the closed-shell algorithms. Moreover, we will show that, unlike existing open-shell perturbation theories, the new methodology can be easily extended to excited states having the same symmetry as the ground state.

\subsection{Open-Shell Perturbation Theory for the Ground State}

\subsubsection{Basic Theory}

The spin-unrestricted formalism for the ROHF functions developed in Sect. 2.2 facilitates the development of a well-defined open-shell many-body Møller-Plesset-like 
perturbation theory. Indeed, the zero-order Hamiltonian can be written as a sum of Fock operators for each electron:

$$
H_{0}^{(0)}=\sum_{k}^{n^{\alpha}} F_{0}^{\alpha}(k)+\sum_{k}^{n^{\beta}} F_{0}^{\beta}(k)
$$

with Fock operators

$$
F_{0}^{\alpha}=\sum_{i}^{M}\left|\varphi_{0 i}^{\alpha}\right\rangle \varepsilon_{0 i}^{\alpha}\left\langle\varphi_{0 i}^{\alpha}\left|, \quad F_{0}^{\beta}=\sum_{i}^{M}\right| \varphi_{0 i}^{\beta}\right\rangle \varepsilon_{0 i}^{\beta}\left\langle\varphi_{0 i}^{\beta}\right|
$$

and the perturbation operator $V=H-H_{0}^{(0)}$. Here the subscript indicates the ground state, i.e. the orbitals and orbital energies are determined from Eqs. (25) and (26).

It is clear that the Slater determinant $\Phi_{0}^{(0)}$ constructed from orbitals (25), (26) and the determinants $\Phi_{0 i}^{a}$, $\Phi_{0 i j}^{a b}$, etc. corresponding to single, double, etc. excitations, obtained by replacing the occupied spin-orbitals by virtual spin-orbitals, form the orthonormal basis set in the many-particle space of states and are eigenfunctions of $H_{0}^{(0)}$, i.e.

$$
\begin{aligned}
& H_{0}^{(0)}\left|\Phi_{0}^{(0)}\right\rangle=E_{0}^{(0)}\left|\Phi_{0}^{(0)}\right\rangle, \quad E_{0}^{(0)}=\left\langle\Phi_{0}^{(0)}\left|H_{0}^{(0)}\right| \Phi_{0}^{(0)}\right\rangle=\sum_{i}^{n^{\alpha}} \varepsilon_{0 i}^{\alpha}+\sum_{i}^{n^{\beta}} \varepsilon_{0 i}^{\beta} \\
& H_{0}^{(0)}\left|\Phi_{0 i}^{a}\right\rangle=E_{0 i}^{a}\left|\Phi_{0 i}^{a}\right\rangle, \quad E_{0 i}^{a}=E_{0}^{(0)}-\varepsilon_{0 i}^{\gamma}+\varepsilon_{0 a}^{\gamma}, \quad \gamma=\alpha, \beta \\
& H_{0}^{(0)}\left|\Phi_{0 i j}^{a b}\right\rangle=E_{0 i j}^{a b}\left|\Phi_{0 i j}^{a b}\right\rangle, \quad E_{0 i j}^{a b}=E_{0}^{(0)}-\varepsilon_{0 i}^{\gamma}-\varepsilon_{0 j}^{\gamma}+\varepsilon_{0 a}^{\gamma}+\varepsilon_{0 b}^{\gamma} .
\end{aligned}
$$

Applying Rayleigh-Schrödinger perturbation theory to the problem (62),(63), it is then easy to show that the sum of the zero-order and first-order energy $E_{0}=E_{0}^{(0)}+E_{0}^{(1)}$ yields the energy expectation value evaluated with respect to the reference function $\Phi_{0}^{(0)}$. Indeed

$$
E_{0}^{(0)}+E_{0}^{(1)}=\left\langle\Phi_{0}^{(0)}\left|H_{0}^{(0)}\right| \Phi_{0}^{(0)}\right\rangle+\left\langle\Phi_{0}^{(0)}|V| \Phi_{0}^{(0)}\right\rangle=\left\langle\Phi_{0}^{(0)}|H| \Phi_{0}^{(0)}\right\rangle .
$$

Note that the reduced resolvent operator

$$
R_{0}^{(0)}=Q_{0}^{(0)}\left(E_{0}^{(0)}-H_{0}^{(0)}\right)^{-1} Q_{0}^{(0)}, \quad Q_{0}^{(0)}=I-\left|\Phi_{0}^{(0)}\right\rangle\left\langle\Phi_{0}^{(0)}\right|
$$

has a diagonal form in the basis set of excited configurations. Therefore, this can be expressed in the form

$$
R_{0}^{(0)}=\sum_{i}^{o c c} \sum_{a}^{\text {virt }} \frac{\left|\Phi_{0 i}^{a}\right\rangle\left\langle\Phi_{0 i}^{a}\right|}{E_{0}^{(0)}-E_{0 i}^{a}}+\sum_{i<j}^{o c c} \sum_{a<b}^{\text {virt }} \frac{\left|\Phi_{0 i j}^{a b}\right\rangle\left\langle\Phi_{0 i j}^{a b}\right|}{E_{0}^{(0)}-E_{0 i j}^{a b}}+\ldots
$$


The summations in this expression are over spin orbitals. We omitted the summations corresponding to the higher order excitations.

Because of the Brillouin theorem, the first-order corrections to the wave functions have the form:

$$
\left|\Phi_{0}^{(1)}\right\rangle=R_{0}^{(0)} V\left|\Phi_{0}^{(0)}\right\rangle=\sum_{i<j}^{o c c} \sum_{a<b}^{v i r t}\left|\Phi_{o i j}^{a b}\right\rangle\left\langle\Phi_{0 i j}^{a b}|H| \Phi_{o i j}^{a b}\right\rangle\left(\varepsilon_{0 i}+\varepsilon_{0 j}-\varepsilon_{0 a}-\varepsilon_{0 b}\right)^{-1} .
$$

The second-order correction to the ground state energy $E_{0}^{(2)}$ is expressed in terms of spin-orbitals and orbital energies:

$$
E_{0}^{(2)}=\sum_{i>j}^{o c c} \sum_{a>b}^{v i r t} \frac{\left|\left(\varphi_{0 a} \varphi_{0 i} \mid \varphi_{0 b} \varphi_{0 j}\right)-\left(\varphi_{0 a} \varphi_{0 j} \mid \varphi_{0 b} \varphi_{0 i}\right)\right|^{2}}{\varepsilon_{0 i}+\varepsilon_{0 j}-\varepsilon_{a}-\varepsilon_{0 b}} .
$$

We emphasise that the summations are over spin-orbitals. $a$ and $b$ are virtual orbitals while $i$ and $j$ are occupied orbitals. The above expression is suitable for practical calculations.

Thus, the orbitals based on asymptotic projection lead to a many-body perturbation theory similar in form to the original to the original Møller-Plesset perturbation theory. In terms of computational cost, this new open-shell perturbation theory, like the OPT1 and OPT2 theories of Murray and Davidson [23] and the ZAPT theory of Lee and Jayatilaka [25], has an obvious advantage over the RMP [21]. The new theory is based on only one set of spatial molecular orbitals whereas the RMP has two sets.

\subsubsection{Application to the Singlet-Triplet Separation in the $\mathrm{CH}_{2}$ Molecule}

There have been a large number of experiments and theoretical studies of the singlettriplet ${ }^{1} A_{1}-{ }^{3} B_{1}$ separation in the $\mathrm{CH}_{2}$ molecule (see, e.g. the work of Sherrill, van Huis, Yamaguchi and Schaefer [63] and also that of Bauschlicher and Taylor [64]) which provide excellent data for assessing new methods. The different spin and spatial symmetry of the states imposes stringent requirements on the methods employed at both the SCF level and in the perturbation theory calculations. We carried out calculations with three basis sets containing $24 s, 28 s$, and $42 s$ Gaussians, respectively. The orbital exponents and positions were determined by minimizing the energy for each individual state. The parameters for our largest basis set of $42 s$ functions can be found in the work of Glushkov [24]. The nuclear coordinates are as follows: the ${ }^{1} A_{1}$ state: $\mathrm{C}(0,0,0) ; \mathrm{H} 1(-1.64403,-1.32213,0)$; $\mathrm{H} 2(1.64403,-1.32213,0)$ and the ${ }^{3} B_{1}$ state: $\mathrm{C}(0,0,0)$; $\mathrm{H} 1(-1.871093,-0.82525,0)$; H2 $(1.871093,-0.82525,0)$.

The energies of the ${ }^{1} A_{1}$ and ${ }^{3} B_{1}$ states at the SCF and second order perturbation theory levels are given in Table 12 together with the corresponding energy splittings. The ${ }^{1} A_{1}$ state is described by a closed-shell determinant and, therefore, the standard restricted Hartree-Fock and the second-order many-body Møller-Plesset perturbation expansion were used. 
Table $12 \mathrm{SCF}$ and second order perturbation theory energies (hartrees) of methelene in the ${ }^{1} A_{1}$ and ${ }^{3} B_{1}$ states and the ${ }^{1} A_{1}-{ }^{3} B_{1}$ separation energy (in $\mathrm{kcal} / \mathrm{mol}$ )

\begin{tabular}{lcccc}
\hline Energy & Basis set & ${ }^{3} B_{1}$ & ${ }^{1} A_{1}$ & $\Delta E\left({ }^{1} A_{1}-{ }^{3} B_{1}\right)$ \\
\hline$E_{S C F}$ & $24 s$ & -38.897795 & -38.858109 & 24.90 \\
& $28 s$ & -38.909868 & -38.872370 & 23.53 \\
& $42 s$ & -38.929603 & -38.889249 & 25.33 \\
& $D Z P[63]$ & -38.927640 & -38.885590 & 26.39 \\
$E_{P T 2^{\mathrm{a}}}$ & $24 s$ & -38.984048 & -38.957602 & 16.59 \\
& $28 s$ & -39.008662 & -38.984938 & 14.88 \\
& $42 s$ & -39.045811 & -39.023562 & 13.96 \\
$C A S P T 2^{\mathrm{b}}$ & $D Z P$ & -39.037660 & -39.013080 & 15.43 \\
$R M P^{\mathrm{c}}$ & $T Z 2 P$ & - & - & 18.05 \\
$O P T 2^{\mathrm{c}}$ & $T Z 2 P$ & - & - & 17.99 \\
$Z A P T^{\mathrm{c}}$ & $T Z 2 P$ & - & -39.027183 & 11.97 \\
$F C I^{\mathrm{d}}$ & $D Z P$ & -39.046260 & & \\
\hline
\end{tabular}

a $-E_{P T 2}=E_{S C F}+E^{(2)}$

$\mathrm{b}$ - CASPT2, second-order of multireference perturbation theory based on a CAS wave function [40].

c - Values were taken from Ref. [25].

d - Full configuration interaction method (FCI) [64].

One can see that our basis set of $42 s$ functions yields SCF energies that are close to those obtained with the DZP basis set. We observe that the value of the energy splitting computed by using our scheme is improved when the size of the basis set increases. A comparison of our asymptotic projection based technique in its secondorder implementation with other open-shell perturbation theories (CASPT2, OPT2, RMP and ZAPT) shows the new method yields values of the singlet-triplet splitting closest to the FCI reference value.

In concluding this section, we note that the present results could be improved by using multireference perturbation theory since the singlet state ${ }^{1} A_{1}$ is known to have two important configurations [64]. An alternative asymptotic projection-based multireference perturbation theory [65] can be used for this.

\subsection{Open-Shell Perturbation Theory for the Excited States}

To our knowledge, there is, at present, no analogue of many-body Møller-Plesset perturbation theory for excited states having the same symmetry as the ground state or some lower lying excited state. The study of such systems often involves the use of a multireference formalism. Such methods are indispensable in studies of systems where single-configuration methods cannot be applied. Nevertheless, it would be very useful to have a perturbation theory formalism the description of excited states 
which can be adequately described by a single Slater determinant. Such an approach shares the computational advantages of the widely used many-body Møller-Plesset perturbation theory. It would be especially useful for calculations of energy differences. In addition, it is important to calculate the ground-state and excited-state energies in a balanced manner, i.e.:

(i) Reference configurations are constructed by employing the same computational scheme. For example, the ground and excited SCF functions are constructed using the Hartree-Fock equations, whose solutions are approximated in oneparticle basis sets adjusted specifically to the state under consideration.

(ii) Correlation effects are taken into account using comparable schemes for the ground- and excited-states, for example, using many-body Møller-Plesset-like perturbation theory.

\subsubsection{Second-Order Correlation Energy for Excited States}

For simplicity, we shall consider the first excited state energy. In this case, the zeroorder Hamiltonian is similar to that for the ground state, but the Fock operators are based on the excited state orbitals and orbital energies from Eqs. (60), i.e.

$$
H^{(0)}=\sum_{k}^{n^{\alpha}} F^{\alpha}(k)+\sum_{k}^{n^{\beta}} F^{\beta}(k)
$$

with Fock operators

$$
F^{\alpha}=\sum_{i}^{M-1}\left|\varphi_{i}^{\alpha}\right\rangle \varepsilon_{i}^{\alpha}\left\langle\varphi_{i}^{\alpha}\left|, \quad F^{\beta}=\sum_{i}^{M-1}\right| \varphi_{i}^{\beta}\right\rangle \varepsilon_{i}^{\beta}\left\langle\varphi_{i}^{\beta}\right| .
$$

In the following, we shall omit the lower subscript for the excited states. The the upper limit of the summations is $M-1$ because the vector $\left|\varphi_{0 n}^{\alpha}\right\rangle$ is excluded from the subspace of virtual molecular orbitals. It is important that singly, $\Phi_{i}^{a}$, doubly, $\Phi_{i j}^{a b}$, etc. excited configurations with respect to an excited state Slater determinant $\Phi^{(0)}$ are eigenfunctions of the Hamiltonian $H^{(0)}(66)$ and they are orthogonal to the ground state Slater determinant $\Phi_{0}^{(0)}$ because $\left\langle\varphi_{0 n}^{\alpha} \mid \varphi_{i}^{\alpha}\right\rangle=0, i=1,2, \ldots, M-1$.

In contrast to the ground state case, for the excited state it is necessary to take into consideration the orthogonality constraints. For the first-order correction to the excited state reference function, $\Phi^{(1)}$, these constraints have the form

$$
\left\langle\Phi^{(0)} \mid \Phi^{(1)}\right\rangle=0
$$

The first-order correction can be written in the following form

$$
\left|\Phi^{(1)}\right\rangle=\left(I-P_{0}^{(0)}\right)\left|\Phi^{(1)}\right\rangle+P_{0}^{(0)}\left|\Phi^{(1)}\right\rangle
$$

where $I$ is the identity operator and $P_{0}^{(0)}=\left|\Phi_{0}^{(0)}\right\rangle\left\langle\Phi_{0}^{(0)}\right|$. 
It should be stressed that $\left|\Phi^{(1)}\right\rangle$ is constructed in the basis of singly, doubly, etc. excited configurations of $\left|\Phi^{(0)}\right\rangle$, which, due to the asymptotic projection method, are orthogonal both to $\left|\Phi^{(0)}\right\rangle$ and $\left|\Phi_{0}^{(0)}\right\rangle$. Therefore, the solution of the first-order equation

$$
\left(H^{(0)}-E^{(0)}\right)\left|\Phi^{(1)}\right\rangle=-\left(V-E^{(1)}\right)\left|\Phi^{(0)}\right\rangle, \quad V=H-H^{(0)}
$$

determines only part of the correction, the projection $\left(I-P_{0}^{(0)}\right)\left|\Phi^{(1)}\right\rangle$ satisfying the condition (68), but does not determine the other part, $P_{0}^{(0)}\left|\Phi^{(1)}\right\rangle$. This projection should be determined by the orthogonality condition for the states in the first-order perturbation theory, i.e.

$$
P_{0}^{(0)}\left|\Phi^{(1)}\right\rangle=-P_{0}^{(1)}\left|\Phi^{(0)}\right\rangle,
$$

where

$$
P_{0}^{(1)}=\left|\Phi_{0}^{(0)}\right\rangle\left\langle\Phi_{0}^{(1)}|+| \Phi_{0}^{(1)}\right\rangle\left\langle\Phi_{0}^{(0)}\right| .
$$

Such a scheme of the construction for $\Phi^{(1)}$ is compatible with both the perturbation theory equations and the orthogonality restrictions (68) and (69).

The final expression for the first-order correction to the excited state reference function takes the form

$$
\left|\Phi^{(1)}\right\rangle=R^{(0)} V\left|\Phi^{(0)}\right\rangle-\left|\Phi_{0}^{(0)}\right\rangle\left\langle\Phi_{0}^{(1)} \mid \Phi^{(0)}\right\rangle
$$

where $R^{(0)}=Q^{(0)}\left(E^{(0)}-H^{(0)}\right)^{-1} Q^{(0)}$ is the reduced resolvent operator, and $Q^{(0)}$ is the orthoprojector onto the complementary space, i.e. $Q^{(0)}=I-\left|\Phi^{(0)}\right\rangle\left\langle\Phi^{(0)}\right|$.

It is well-known that the Rayleigh-Schrödinger perturbation theory leads to the following expression for the second order correction to the energy

$$
E^{(2)}=\left\langle\Phi^{(0)}|H| \Phi^{(1)}\right\rangle
$$

or, taking (70) into account, we have the expression

$$
E^{(2)}=\sum_{i>j}^{o c c} \sum_{a>b}^{\text {virt }} \frac{\left|\left(\varphi_{a} \varphi_{i} \mid \varphi_{b} \varphi_{j}\right)-\left(\varphi_{a} \varphi_{j} \mid \varphi_{b} \varphi_{i}\right)\right|^{2}}{\varepsilon_{i}+\varepsilon_{j}-\varepsilon_{a}-\varepsilon_{b}}-\left\langle\Phi^{(0)}|H| \Phi_{0}^{(0)}\right\rangle\left\langle\Phi_{0}^{(1)} \mid \Phi^{(0)}\right\rangle
$$

The first term in Eq. (71) is immediately recognized as the second-order perturbation theory expression for the ground state energy ( $c f$. with (65)). Single excitations do not contribute because the excited state orbitals, like the ground state orbitals, satisfy the generalized Brillouin theorem. The second term in Eq. (71) appears because the Hartree-Fock ground and excited state functions are not eigenfunctions of the Hamiltonian $H$. In practice, if the ground state and excited state energies and the corresponding wave functions are known accurately then the coupling matrix element $\left\langle\Phi_{0}|H| \Phi_{1}\right\rangle$ is expected to be small (see also paper I [27], Sect. 3.1). Furthermore, as the overlap element $\left\langle\Phi_{0}^{(1)} \mid \Phi^{(0)}\right\rangle<1$, then during the first stage of calculations the last term in Eq. (71) may be neglected. 
Thus, we obtain comparable perturbation schemes for the ground and excited state energies. Use of the asymptotic projection technique ensures that calculations for excited states require practically the same computational time as those for the ground state.

\subsubsection{Numerical Examples}

Below we demonstrate some possibilities of the single reference-based perturbation theory based on orbitals obtained by asymptotic projection for calculations of the total energies and excitation energies of the $\mathrm{HeH}$ and $\mathrm{BeF}$ molecules. We studied the $X^{2} \Sigma^{+}, A^{2} \Sigma^{+}, C^{2} \Sigma^{+}$, and $D^{2} \Sigma^{+}$states of $\mathrm{HeH}$ and the $X^{2} \Sigma^{+}, B^{2} \Sigma^{+}$and $C^{2} \Sigma^{+}$ states of the $\mathrm{BeF}$ molecule.

For $\mathrm{HeH}$, the basis set of $18 s$ Gaussians employed in Sect. 2.2.2 was extended to a $18 s 3 p$ set, i.e. $18 s$ functions were distributed along the molecular axis ( $z$-axis) with the basis set parameters determined by minimizing the total SCF energy for each individual state. It should be noted that such basis set extension does not modify the subspace of occupied orbitals nor, therefore, the SCF energy, but it facilitates and improved description of correlation effects. The orbital exponents and positions of the $p$-functions ( $p_{x}$ and $p_{y}$ ) were determined by using Hylleraas' variational principle [66]. This allowed us to minimize the error associated with truncation of one-particle basis sets and, thus, to assess more precisely the errors of the method itself. In addition, each $p$ function $\left(p_{x}, p_{y}\right)$ was represented by a linear combination of two s-functions, i.e. the so-called Gaussian lobe representation [67] was used.

The results of our calculations of the total energies and excitation energies are presented in Table 13 where they are compared to those obtained by the CI method [62] and experimental data.

The BeF molecule was studied in detail in [68] where its various properties were determined using the CI method. Experimental data are also available for this molecule [69]. The best results in [68] were obtained with the mixed one-particle basis set consisting of the Slater and two-center functions. We carried out calculations with the basis sets consisting of $24 s$ Gaussian functions for the $X^{2} \Sigma^{+}, B^{2} \Sigma^{+}$

Table 13 Excited state energies (hartrees) and excitation energies $(\Delta E, \mathrm{eV})$ from the $A^{2} \Sigma^{+}$state of $\mathrm{HeH}$ at the different levels of approximation at $R=1.5 \mathrm{bohr}$

\begin{tabular}{lccc}
\hline Method & $A^{2} \Sigma^{+}$ & $B^{2} \Sigma^{+}$ & $D^{2} \Sigma^{+}$ \\
\hline Hartree-Fock-asymptotic projection & -3.066606 & -3.014785 & -2.988232 \\
$E^{(2)}$ & -0.033363 & -0.029915 & -0.030027 \\
$E_{M P 2}$ & -3.099969 & -3.044700 & -3.018259 \\
$E_{C I}[62]$ & -3.112706 & -3.055797 & -3.030025 \\
$\Delta E_{H F}$ & 0 & 1.41 & 2.13 \\
$\Delta E_{M P 2}$ & 0 & 1.50 & 2.22 \\
$\Delta E_{C I}[62]$ & 0 & 1.53 & 2.25 \\
$\Delta E_{\text {exp }}[62]$ & 0 & 1.55 & 2.26 \\
\hline
\end{tabular}


states and $26 s$ functions for the $C^{2} \Sigma^{+}$state and $4 p$-functions $\left(4 p_{x}\right.$ and $\left.4 p_{y}\right)$, which, in turn, were represented by the lobe approximated using lobes functions as we did for $\mathrm{HeH}$. These $p$-functions simulated the behavior of $1 \pi_{x}$ and $1 \pi_{y}$ orbitals in the $1 \sigma_{2} 1 \sigma_{2} 1 \sigma_{2} 1 \sigma_{2} 1 \pi_{4} \phi$ configurations, where $\phi=5 \sigma, 6 \sigma$ and $7 \sigma$ correspond to the $X^{2} \Sigma^{+}, B^{2} \Sigma^{+}$and $C^{2} \Sigma^{+}$states, respectively. The calculations were performed at internuclear distance $R=2.5 \mathrm{bohr}$, which is close to the equilibrium separation for all the states under consideration. In this case, the basis set parameters both of $s$-functions and $p$-functions were determined for each individual state by minimizing the corresponding energy in the single determinant approximation. Such an optimization of the restricted basis sets is very important for the excited states, and its contribution to the total energy is comparable with the second-order correlation correction as can be seen in Table 14, where the zero-, first- and second-order energies are given. The energies in the column headed A were computed with basis sets optimized for the state under study whereas the energies presented in column $\mathrm{B}$ were obtained with the basis set adjusted to the ground state $\left(X^{2} \Sigma^{+}\right)$. In addition, a comparison of columns A and B also shows that the correlation energy (the row $\left.E^{(2)}\right)$ depends slightly on the basis set optimization.

Of course, the basis sets employed are not of sufficient size to approach the complete basis limit and thus the comparison of absolute values with more precise ones is not meaningful. However, if basis set optimization has been carried out for each individual state and the similar scheme for accounting the correlation effects has been used (Møller-Plesset like perturbation theory in our case), then energy contributions from incompleteness of basis set may be assumed to be similar for adjacent excited states and, therefore, comparisons of relative positions of energy levels computed against more precise or experimental ones are instructive to estimate the performance of the method. We can see from Table 15 that the method introduced in this paper yield reasonable excitation energies which are closer to experimental results [69] than the CI values obtained in Ref. [68].

Table 14 The total energies (hartrees) of the BeF molecule calculated in different orders of perturbation theory at $R=2.5 \mathrm{bohr}$

\begin{tabular}{lrrrrr}
\hline Order of perturbation & $A\left(X^{2} \Sigma^{+}\right)$ & $A\left(B^{2} \Sigma^{+}\right)$ & $B\left(B^{2} \Sigma^{+}\right)$ & $A\left(C^{2} \Sigma^{+}\right)$ & $B\left(C^{2} \Sigma^{+}\right)$ \\
\hline$E^{(0)}$ & -69.2212 & -70.8649 & -68.9800 & -70.2098 & -68.6674 \\
$E^{(0)}+E^{(1)}$ & -114.1036 & -113.8823 & -113.7909 & -113.8751 & -113.5953 \\
$E^{(2)}$ & -0.1743 & -0.1725 & -0.1730 & -0.1690 & -0.1684 \\
$E_{M P 2}$ & -114.2779 & -114.0548 & -113.9647 & -114.0441 & -113.7637 \\
\hline
\end{tabular}

Table 15 Excitation energies $(\Delta E, \mathrm{eV})$ from the $X^{2} \Sigma^{+}$state of $\mathrm{BeF}$ at the different levels of approximation at $\mathrm{R}=2.5 \mathrm{bohr}$

\begin{tabular}{lcccc}
\hline State & $\begin{array}{c}\text { Hartree-Fock-asymptotic } \\
\text { projection }\end{array}$ & MP2 & CI [68] & Experiment [69] \\
\hline$B^{2} \Sigma^{+}$ & 6.02 & 6.07 & 6.25 & 6.12 \\
$B^{2} \Sigma^{+}$ & 6.22 & 6.36 & 6.69 & 6.24 \\
\hline
\end{tabular}




\section{Conclusions}

We have presented a detailed description of open-shell SCF theory for the ground and excited states based on an easily implemented asymptotic projection method for taking the orthogonality constraints into account in eigenvalue problems which was proposed earlier. The effectiveness of such a SCF-asymptotic projection theory and its performance have been demonstrated:

(i) By solving the long-standing problem of off-diagonal Lagrange multipliers in open-shell self-consistent theory. We have considered an alternative to the Roothaan's open-shell technique that does not involve off-diagonal Lagrange multipliers. We have constructed a well-defined perturbation theory based on this technique which can be used to account for correlation effects.

(ii) We have given an example of the variational determination of excited electronic states having the same spatial and spin symmetry as the ground state. The results given above in $(i)$ have thus been extended to excited state self-consistent theory and an analogue of the many-body Møller-Plesset perturbation theory for excited states has been developed.

Finally, it is worth pointing out the similarity between the SCF-asymptotic projection formalism developed here and the Optimized Effective Potential method for practical excited state calculations within density functional theory. Preliminary results can be found in the work of Glushkov, of Glushkov and Levy and of Glushkov and Gidopoulos [70-72].

Acknowledgment V.N.G. thanks Prof. A. Theophilou for useful and stimulating discussions during the early stages of this work.

\section{References}

1. C.C.J. Roothaan, Rev. Mod. Phys. 32, 179, 1960.

2. S. Huzinaga, Phys. Rev. 120, 866, 1960.

3. F.W. Birss and S. Fraga, J. Chem. Phys. 38, 2552, 1963.

4. E.R. Davidson, Chem. Phys. Lett. 21, 565, 1963.

5. J.P. Dahl, H. Johanson, D.R. Truax and T. Zeiger, Chem. Phys. Lett. 6, 64, 1970.

6. V.A. Kuprievich and O.V. Shramko, Int. J. Quantum. Chem. 6, 327, 1972.

7. K. Hirao and H. Nakatsuji, J. Chem. Phys. 59, 1457, 1973.

8. K. Hirao, J. Chem. Phys. 60, 3215, 1974.

9. D. Peters, J. Chem. Phys. 57, 4351, 1972.

10. R. Albat and N. Gruen, Chem. Phys. Lett. 18, 572, 1973.

11. R. McWeeny, Chem. Phys. Lett. 35, 13, 1975.

12. R. McWeeny and B.T. Sutcliffe, Methods of Molecular Quantum Mechanics, Academic Press, New York, 1976.

13. R. McWeeny and G.H.F. Diercksen, J. Chem. Phys. 49, 4852, 1968.

14. R. Carbo and J.M. Riera, in Lecture Notes in Chemistry v.5, G. Berthier, ed., Springer-Verlag, Berlin, 1978. 
15. M.J. Frisch, G.W. Trucks, H.B. Schlegel, G.E. Scuseria, M.A. Robb, J.R. Cheeseman, J.A Montgomery, Jr., T. Vreven, K.N. Kudin, J.C. Burant, J.M. Millam, S.S. Iyengar, J. Tomasi, V. Barone, B. Mennucci, M. Cossi, G. Scalmani, N. Rega, G.A. Petersson, H. Nakatsuji, M. Hada, M. Ehara, K. Toyota, R. Fukuda, J. Hasegawa, M. Ishida, T. Nakajima, Y. Honda, O. Kitao, H. Nakai, M. Klene, X. Li, J.E. Knox, H.P. Hratchian, J.B. Cross, V. Bakken, C. Adamo, J. Jaramillo, R. Gomperts, R.E. Stratmann, O. Yazyev, A.J. Austin, R. Cammi, C. Pomelli, J.W. Ochterski, P.Y. Ayala, K. Morokuma, G.A. Voth, P. Salvador, J.J. Dannenberg, V.G. Zakrzewski, S. Dapprich, A.D. Daniels, M.C. Strain, O. Farkas, D.K. Malick, A.D. Rabuck, K. Raghavachari, J.B. Foresman, J.V. Ortiz, Q. Cui, A.G. Baboul, S. Clifford, J. Cioslowski, B.B. Stefanov, G. Liu, A. Liashenko, P. Piskorz, I. Komaromi, R.L. Martin, D.J. Fox, T. Keith, M.A. Al-Laham, C.Y. Peng, A. Nanayakkara, M. Challacombe, P.M.W. Gill, B. Johnson, W. Chen, M.W. Wong, C. Gonzalez and J. A. Pople, Gaussian 03, Revision C.02, Gaussian, Inc., Wallingford CT, 2004.

16. M.W. Schmidt, K.K. Baldridge, J.A. Boatz, S.T. Elbert, M.S. Gordon, J.H. Jensen, S. Koseki, N. Matsunaga, K.A. Nguyen, S. Su, T.L. Windus, M. Dupuis and J.A. Montgomery J. Comput. Chem., 14, 1347-1363(1993); M.S. Gordon and M.W. Schmidt, in Theory and Applications of Computational Chemistry: the first forty years, edited by C.E. Dykstra, G. Frenking, K.S. Kim, G.E. Scuseria, Elsevier, Amsterdam, pp. 1167-1189, 2005.

17. B.N. Plakhutin and E.V. Gorelik, Breslavskaya, J. Chem. Phys. 125, 204110, 2006.

18. I. Hubač and P. Čársky, Phys. Rev. A22, 2392, 1980.

19. S. Wilson, Theoret. chim. Acta. 61, 343, 1984.

20. J.S. Andrews, D. Jayatilaka, R.G.A. Bone, N.C. Handy, and R.D. Amos, Chem. Phys. Lett. 183, 423, 1991

21. P.J. Knowles, J.S. Andrews, R.D. Amos, N.C. Handy and J.A. Pople, Chem. Phys. Lett. 186, 130, 1991.

22. W.J. Lauderdale, J.F. Stanton, J. Gauss, J.D. Watts and R.J. Bartlett, Chem. Phys. Lett. 187, 21, 1991.

23. C. Murray and E.R. Davidson, Chem. Phys. Lett. 187, 451, 1991.

24. V.N. Glushkov, Int. J. Quantum. Chem. 99, 236, 2004.

25. T.J. Lee and D. Jayatilaka, Chem. Phys. Lett. 201, 1, 1993.

26. P.J. Knowles and N.C. Handy, J. Phys. Chem. 92, 3097, 1988.

27. V.N. Glushkov, N.I. Gidopoulos and S. Wilson, this volume.

28. V.N. Glushkov and A.Ya. Tsaune, Zh. Vychisl. Mat. Mat. Fiz. 25, 298, 1985; USSR Comp. Mathemat. Math. Physics, 25, 196, 1986.

29. V.N. Glushkov, J. Math. Chem. 31, 91, 2002

30. V.N. Glushkov, Opt. Spectrosc. 93, 11, 2002.

31. A.Ya. Tsaune, V.N. Glushkov and A.I. Aprashukhin, J. Mol. Struct. (THEOCHEM) 312, 289, 1994.

32. V.N. Glushkov and A.Ya. Tsaune, Chem. Phys. Lett. 262, 59, 1996.

33. V.N. Glushkov, Chem. Phys. Lett. 273, 122, 1997.

34. V.N. Glushkov, Chem. Phys. Lett. 287, 189, 1998.

35. N.I. Gidopoulos, V.N. Glushkov and S. Wilson, Proc. R. Soc. Lond. A. 457, 1657, 2002.

36. V.N.Glushkov and S. Wilson, in: Recent Advances in the Theory of Chemical and Physical Systems, edited by J.-P. Julien et al., p. 107, Springer, Dordrecht, 2006.

37. V.N. Glushkov and A.Ya. Tsaune, Opt. Spectrosc. 87, 267, 1999.

38. V.N. Glushkov and A.Ya. Tsaune, Opt. Spectrosc. 101, 516, 2006.

39. H. Shull and P.-O. Löwdin, Phys. Rev. 1958, 110, 1466, 1958.

40. K. Andersson and B.O. Roos, in: Modern Electronic Structure Theory, Part II, edited by D. Yarkony, pp. 55-109, World Scientific, Singapore, 1995.

41. V.A. Fock, Z. Exsp. Teor. Fiz. 10, 961, 1940.

42. V.N. Glushkov and S. Wilson, Int. J. Quantum. Chem. 89, 237, 2002.

43. V.N. Glushkov, Opt. Spectrosc. 100, 807, 2006.

44. V.N. Glushkov and S. Wilson, Int. J. Quantum. Chem. 99, 903, 2004.

45. K. Morokuma and S. Iwata, Chem. Phys. Lett. 16, 195, 1972. 
46. R. McWeeny, Molec. Phys. 28, 1273, 1974.

47. J. Mrozek and A, Golebiewski, Int. J. Quant. Chem. 12, 207, 1977.

48. E.R. Davidson and L.Z. Stenkamp, Int. J. Quant. Chem. (Symp). 10, 21, 1976.

49. E.R. Davidson and E.L. McMurchie. in Excited States 1 5, 1, 1985.

50. R. Colle, A. Fortunelli and O. Salvetti, Theor. Chim. Acta 71, 467, 1987.

51. N.I. Gidopoulos and A. Theophilou, Phil. Mag. 69, 1067, 1994.

52. A. Theophilou, J. Phys. C 12, 5419, 1979.

53. N.I. Gidopoulos, P.G. Papaconstantinou and E.K.U. Gross, Phys. Rev. Lett. 88, 33003, 2002.

54. N.I. Gidopoulos, P.G. Papaconstantinou and E.K.U. Gross, Physica B: 318, 328, 2002.

55. V.N. Glushkov and A. Theophilou, Phys. Rev. A 64, 064501, 2001.

56. V.N. Glushkov and A. Theophilou, J. Phys. B: At. Mol. Opt. Phys. 35, 2313, 2002.

57. M. Cohen and P.S. Kelly, Can. J. Phys. 43, 1867, 1965.

58. H. Tatewaki, T. Koga, Y. Sakai and A.J. Thakkar, J. Chem. Phys. 101, 4945, 1994.

59. Ch. Froese, J. Chem. Phys. 47, 4010, 1967.

60. M.W. Schmidt and K. Ruedenberg, J. Chem. Phys. 71, 3951, 1979.

61. E.S. Kryachko and S. Wilson, Int. J. Quant. Chem. 93, 112, 2003.

62. I.D.Petsalakis, G.Theodorakopoulos, C.A. Nicolaides and R.J.Buenker, J. Phys. B: At. Mol. Phys. 20, 2339, 1987; 20, 5959, 1987.

63. C.D. Sherrill, T.J. van Huis, Y. Yamaguchi and H.F. Schaefer III, J. Mol. Struct. (THEOCHEM) 400, 139, 1997.

64. C.W. Bauschlicher, Jr. and P.R. Taylor, J. Chem. Phys. 85, 6510, 1986.

65. V.N. Glushkov, Chem. Phys. Lett. 244, 1, 1995.

66. E.A. Hylleraas, Z. Phys. 65, 209, 1930.

67. J. Harisson, J. Chem. Phys. 46, 1115, 1967.

68. Stepanov N.F., Nemukhin A.V., Khrustov V.F. and Safonov A.A. in: Stroenie molekul, Moscow State Univ., p. 229-245, 1986.

69. K.P. Huber and G. Herzberg, Molecular Spectra and Molecular Structure: IV Constants of Diatomic Molecules, Van Nostrand Reinhold, New York, 1979.

70. V.N. Glushkov, Opt. Spectrosc. 99, 684, 2005.

71. V.N. Glushkov and M. Levy, J. Chem. Phys. 126, 174106, 2007.

72. V.N. Glushkov and N.I. Gidopoulos, Int. J. Quant. Chem. 107, 2604, 2007. 